Research Article

\title{
Multiplicative Zagreb Indices of Molecular Graphs
}

\author{
Xiujun Zhang $\mathbb{D}^{1},{ }^{1}$ H. M. Awais $\mathbb{D},^{2}$ M. Javaid $\mathbb{D}^{2},{ }^{2}$ and Muhammad Kamran Siddiqui ${ }^{3}$ \\ ${ }^{1}$ School of Information Science and Engineering, Chengdu University, Chengdu 610106, China \\ ${ }^{2}$ Department of Mathematics, School of Science, University of Management and Technology (UMT), \\ Lahore 54770, Pakistan \\ ${ }^{3}$ Department of Mathematics, COMSATS University Islamabad, Lahore Campus, Islamabad, Pakistan
}

Correspondence should be addressed to M. Javaid; javaidmath@gmail.com

Received 1 November 2019; Accepted 19 November 2019; Published 6 December 2019

Academic Editor: Juan L. G. Guirao

Copyright ( 2019 Xiujun Zhang et al. This is an open access article distributed under the Creative Commons Attribution License, which permits unrestricted use, distribution, and reproduction in any medium, provided the original work is properly cited.

\begin{abstract}
Mathematical modeling with the help of numerical coding of graphs has been used in the different fields of science, especially in chemistry for the studies of the molecular structures. It also plays a vital role in the study of the quantitative structure activities relationship (QSAR) and quantitative structure properties relationship (QSPR) models. Todeshine et al. (2010) and Eliasi et al. (2012) defined two different versions of the 1st multiplicative Zagreb index as $\prod(\Gamma)=\prod_{p \in V(\Gamma)}\left[d_{\Gamma}(p)^{2}\right]$ and $\prod_{1}(\Gamma)=\prod_{p q \in E(\Gamma)}\left[d_{\Gamma}(p)+d_{\Gamma}(q)\right]$, respectively. In the same paper of Todeshine, they also defined the 2nd multiplicative Zagreb index as $\prod_{2}(\Gamma)=\prod_{p q \in E(\Gamma)}\left[d_{\Gamma}(p) \times d_{\Gamma}(q)\right]$. Recently, Liu et al. [IEEE Access; $\left.7(2019) ; 105479--105488\right]$ defined the generalized subdivision-related operations of graphs and obtained the generalized F-sum graphs using these operations. They also computed the first and second Zagreb indices of the newly defined generalized F-sum graphs. In this paper, we extend this study and compute the upper bonds of the first multiplicative Zagreb and second multiplicative Zagreb indices of the generalized F-sum graphs. At the end, some particular results as applications of the obtained results for alkane are also included.
\end{abstract}

\section{Introduction}

The numerical demonstration of a molecular graph can be assumed as a single number, commonly known as topological index (TI). There are many interesting and significant results about TIs to study the different properties of chemical compounds such as chromatographic retention times, heat of formation and evaporation, flash point, viscosity, freezing, boiling and melting point, octanol-water partition coefficient, surface tension, stability, temperature, density, weight, polarizability, connectivity, and solubility. Many medicines, crystalline and nanomaterials, that are used in numerous pharmaceutical industries are examined with the assistance of different TIs, see [1-7]. TIs also study QSPR and QSAR models that join molecular graphs to their molecular characteristics by means of statistical tools. For additional information, see [8-17].
In 1947, to investigate the paraffin's boiling point, Wiener utilized the distance-based TI [18]. Gutman and Trinajstić [19] determined a pair of degree-based first and second Zagreb indices. After this, different impressive TIs are introduced in molecular graph theory [20,21], but the degree-based TIs are famous than others, see [22].

In graph theory, the various operations on different graphs show an important role in the creation of advanced families of graphs, see [23,24]. Yan et al. [13] gave the idea of four operations $S_{1}, R_{1}, Q_{1}$, and $T_{1}$ of graphs and computed the Wiener index of the resultant graphs obtained by using these operations. Eliasi and Taeri [25] introduced the $F_{1}$-sum graphs $\left(\Gamma_{1+F_{1}} \Gamma_{2}\right)$ by using the cartesian product of graphs $F_{1}\left(\Gamma_{1}\right)$ and $\Gamma_{2}$, where $\Gamma_{1}$ and $\Gamma_{2}$ are the two simple graphs and $F_{1}\left(\Gamma_{1}\right)$ is obtained by using $F_{1} \in\left\{S_{1}, R_{1}, Q_{1}, \& T_{1}\right\}$. They also determined the Wiener index of these $F_{1}$-sum graphs. Additionally, Deng et al. [26], Imran and Akhtar [27], Shirdel et al. [28], and Liu et al. [29] determined the 1st and 2nd Zagreb 
indices, F-index, Hyper-Zagreb index, and the 1st general Zagreb index of $F_{1}$-sum graphs.

Recently, Liu et al. [30] defined the generalized form of the aforesaid four operations $\left\{S_{k}, R_{k}, Q_{k}\right.$, and $T_{k}$, for $k \geq 1$ integer $\}$. They also constructed the generalized F-sum graphs $\left(\Gamma_{1+F_{k}} \Gamma_{2}\right.$ for $\left.F_{k} \in\left\{S_{k}, R_{k}, Q_{k}, \& T_{k}\right\}\right)$ and obtained the 1st and 2 nd Zagreb indices. In this paper, we calculate the upper bounds of the 1 st and 2 nd multiplicative Zagreb indices of the $F_{k}$-sum graphs. The remaining article is organized as follows: Section 2 contains few definitions and terminologies, Section 3 covers the main results, and Section 4 includes applications and closing comments.

\section{Preliminaries}

A molecular graph $\Gamma=(V(\Gamma), E(\Gamma))$ has the node (vertex) set $V(\Gamma)=\left\{p_{1}, p_{2}, p_{3}, \ldots, p_{n}\right\}$ and the edge set $E(\Gamma) \subseteq V(\Gamma) \times V(\Gamma)$. The vertices in the molecular graphs are denoted as atoms, and bonds are denoted as edges. The order and size of a chemical structure is denoted as $|V(\Gamma)|=$ $\mu$ and $|E(\Gamma)|=\omega$. The degree $\left(d_{\Gamma}(p)\right)$ is the total edges that are incident on a node $p$. Throughout the paper, we study the finite, indirected, simple (without loops, isolated vertices, and multiple edges), and connected graphs. The well-known TIs are discussed as follows.

Definition 1. Let $\Gamma$ be a graph, then the first and second Zagreb indices are defined as follows:

$$
\begin{aligned}
& M_{1}(\Gamma)=\sum_{p q \in E(\Gamma)}\left[d_{\Gamma}(p)+d_{\Gamma}(q)\right], \\
& M_{2}(\Gamma)=\sum_{p q \in E(\Gamma)}\left[d_{\Gamma}(p) \times d_{\Gamma}(q)\right] .
\end{aligned}
$$

Gutman and Trinajstić [19] defined these indices which are utilized to determine the structural base different characteristics of graphs like molecular complexity, energy, ZE-isomerism, chirality, connectivity, and heterosystems, as well as branching, see [15,31-36].

Definition 2. Let $\Gamma$ be a graph, then the first and second multiplicative Zagreb indices are

$$
\begin{aligned}
& \prod_{1}(\Gamma)=\prod_{p q \in E(\Gamma)}\left[d_{\Gamma}(p)+d_{\Gamma}(q)\right], \\
& \prod_{2}(\Gamma)=\prod_{p q \in E(\Gamma)}\left[d_{\Gamma}(p) \times d_{\Gamma}(q)\right] .
\end{aligned}
$$

During the past two decades, various noteworthy applications regarding multiplicative Zagreb indices have been explained in detail, see [37-46] and the references cited therein. The resultant graphs under the four new generalized subdivision operations defined in [30] are given as follows: (i) $S_{k}(\Gamma)$ is the generalized subdivided graph $(\Gamma)$.

(ii) $R_{k}(\Gamma)$ is the generalized semitotal (point) graph.

(iii) $Q_{k}(\Gamma)$ is the generalized semitotal (line) graph.

(iv) $T_{k}(\Gamma)$ is the generalized total graph. For more details, see Figure 1.

Definition 3. Let $\Gamma_{1}$ and $\Gamma_{2}$ be two graphs, then $F_{k} \in\left\{S_{k}, R_{k}, Q_{k}, T_{k}\right\}$ and $F_{k}\left(\Gamma_{1}\right)$ be a new graph gained after using $F_{k}$ on $\Gamma_{1}$ having an edge set $E\left(F_{k}\left(\Gamma_{1}\right)\right)$ and vertex set $V\left(F_{k}\left(\Gamma_{1}\right)\right)$. So, the generalized $F$-sum graph $\left(\Gamma_{1+F_{k}} \Gamma_{2}\right)$ is a graph having the vertex set $V\left(\Gamma_{1+F_{k}} \Gamma_{2}\right)=V\left(F_{k}\left(\Gamma_{1}\right)\right) \times$ $V\left(\Gamma_{2}\right)=V\left(\Gamma_{1}\right) \cup E\left(\Gamma_{1}\right) \times V\left(\Gamma_{2}\right)$ in such a way that two vertices $\left(p_{1}, q_{1}\right)$ and $\left(p_{2}, q_{2}\right)$ of $V\left(\Gamma_{1+F_{k}} \Gamma_{2}\right)$ are adjacent if and only if $\left[p_{1}=p_{2} \in V\left(\Gamma_{1}\right)\right.$ and $\left.\left(q_{1}, q_{2}\right) \in E\left(\Gamma_{2}\right)\right]$ or $\left[q_{1}=q_{2}\right.$ $\in \Gamma_{2}$ and $\left.\left(p_{1}, p_{2}\right) \in E\left(F_{k}\left(\Gamma_{1}\right)\right)\right]$.

Thus, the generalized $F$-sum graph $\Gamma_{1+F_{k}} \Gamma_{2}$ contains $\left|V\left(\Gamma_{2}\right)\right|$ copies of new graphs $F_{k}\left(\Gamma_{1}\right)$ that are labeled with the vertex set of $\Gamma_{2}$. For further details, see Figures 2 and 3 .

\section{Main Results}

Now, we calculate the key results of the multiplicative Zagreb indices for the different classes on graphs.

Theorem 1. Let $\Gamma_{1}, \Gamma_{2}$ be two graphs with $\left|V\left(\Gamma_{1}\right)\right|=\mu_{1}$, $\left|V\left(\Gamma_{2}\right)\right|=\mu_{2}, \quad\left|E\left(\Gamma_{1}\right)\right|=\omega_{1}$, and $\left|E\left(\Gamma_{2}\right)\right|=\omega_{2}$. For $\mu_{1}, \mu_{2} \geq 4$ and and $k \geq 1$, we have

$$
\begin{aligned}
\prod_{1}\left(\Gamma_{1}+{ }_{S_{k}} \Gamma_{2}\right) \leq & \frac{1}{\mu_{1} \omega_{2}}\left[4 \omega_{1} \omega_{2}+\mu_{1} M_{1}\left(\Gamma_{2}\right)\right]^{\mu_{1} \omega_{2}} \\
& \times \frac{1}{2 \omega_{1} \mu_{2}}\left[4 \omega_{1} \omega_{2}+\mu_{2} M_{1}\left(S_{1}\left(\Gamma_{1}\right)\right)\right]^{2 \omega_{1} \mu_{2}} \\
& \times \frac{1}{\omega_{1} \mu_{2}(k-1)}\left[4(k-1) \mu_{2} \omega_{1}\right]^{\omega_{1} \mu_{2}(k-1)}, \\
\prod_{2}\left(\Gamma_{1}+{ }_{S_{k}} \Gamma_{2}\right) \leq & \frac{1}{\mu_{1} \omega_{2}}\left[\omega_{2} M_{1}\left(\Gamma_{1}\right)+2 \omega_{1} M_{1}\left(\Gamma_{2}\right)+\mu_{1} M_{2}\left(\Gamma_{2}\right)\right]^{\mu_{1} \omega_{2}} \\
& \times \frac{1}{2 \mu_{2} \omega_{1}}\left[\mu_{2} M_{2}\left(S_{1}\left(\Gamma_{1}\right)\right)+8 \omega_{1} \omega_{2}\right]^{2 \mu_{2} \omega_{1}} \\
& \times \frac{1}{(k-1) \mu_{2} \omega_{1}}\left[4(k-1) \mu_{2} \omega_{1}\right]^{(k-1) \mu_{2} \omega_{1}} .
\end{aligned}
$$

Proof

(a) Consider $d(p, q)=d_{\Gamma_{1+s_{k}}} \Gamma_{2}(p, q)$ as a degree of a node $(p, q)$ in $\Gamma_{1}+{ }_{s_{k}} \Gamma_{2}$. 


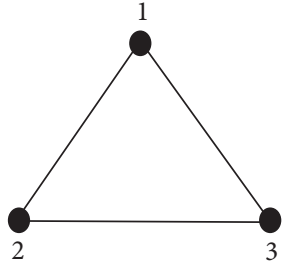

(a)

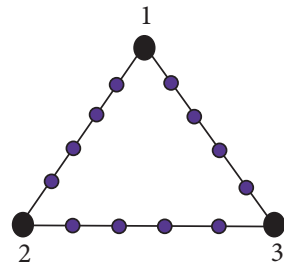

(b)

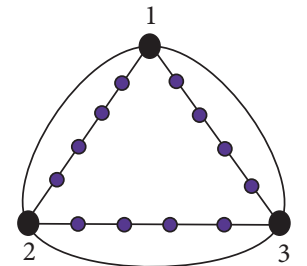

(c)

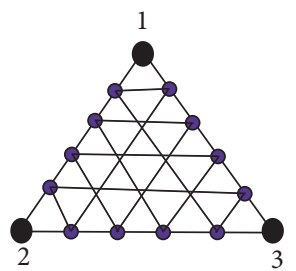

(d)

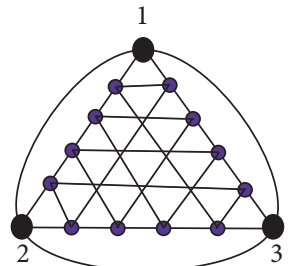

(e)

Figure 1: (a) $\Gamma$, (b) $S_{4}(\Gamma)$, (c) $R_{4}(\Gamma)$. (d) $Q_{4}(\Gamma)$, and (e) $T_{4}(\Gamma)$.

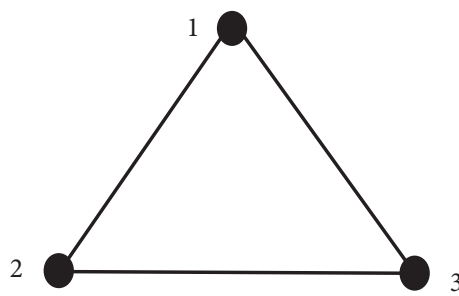

(a)

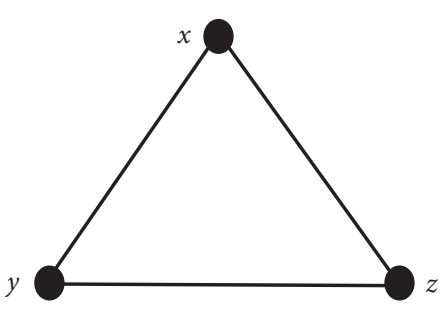

(b)

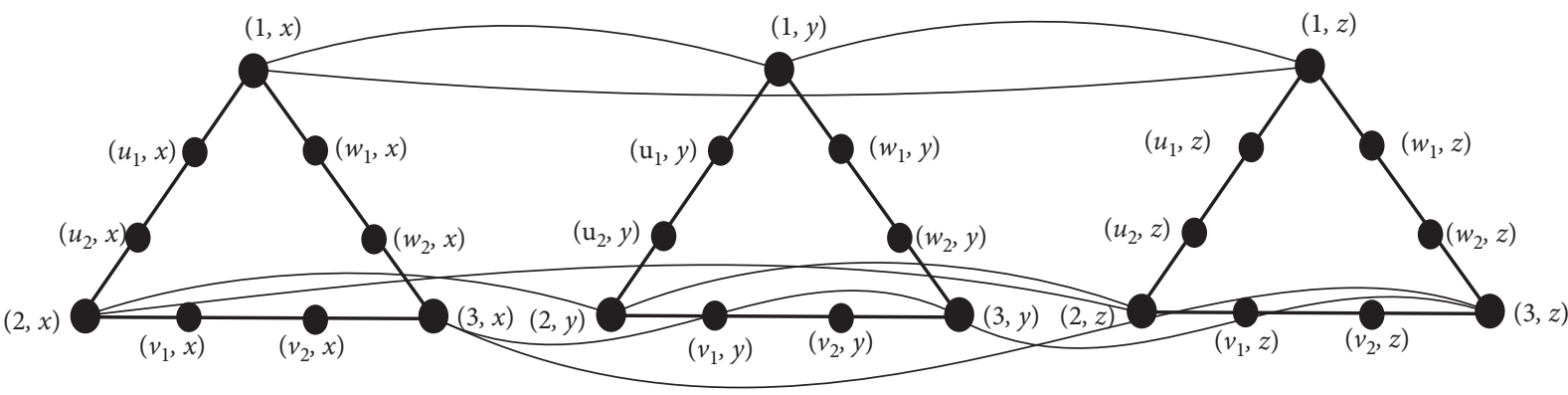

(c)

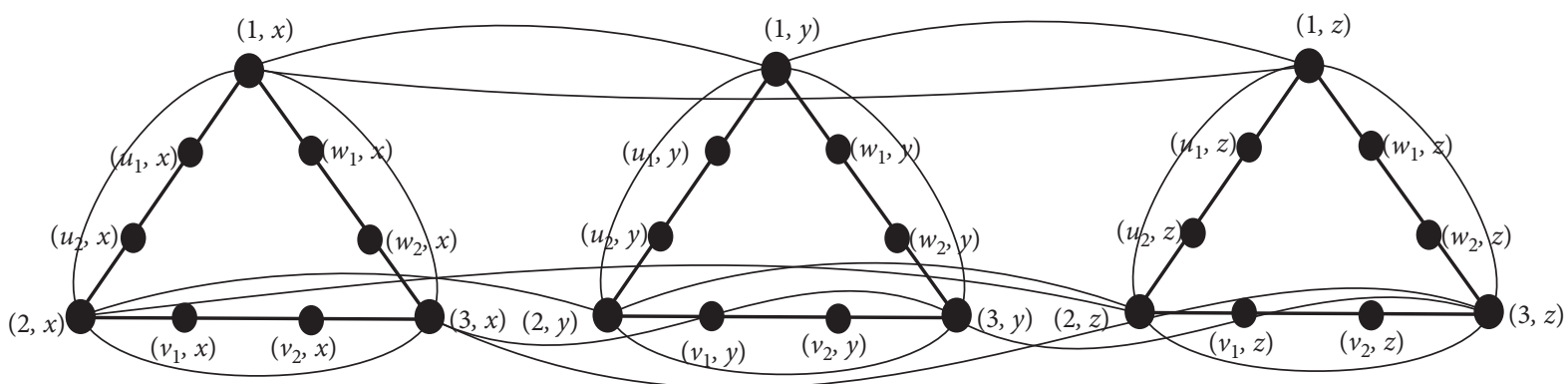

(d)

Figure 2: (a) $\Gamma_{1} \cong C_{3}$, (b) $\Gamma_{2} \cong C_{3}$, (c) $\Gamma_{1+S_{2}} \Gamma_{2}$, and (d) $\Gamma_{1+R_{2}} \Gamma_{2}$.

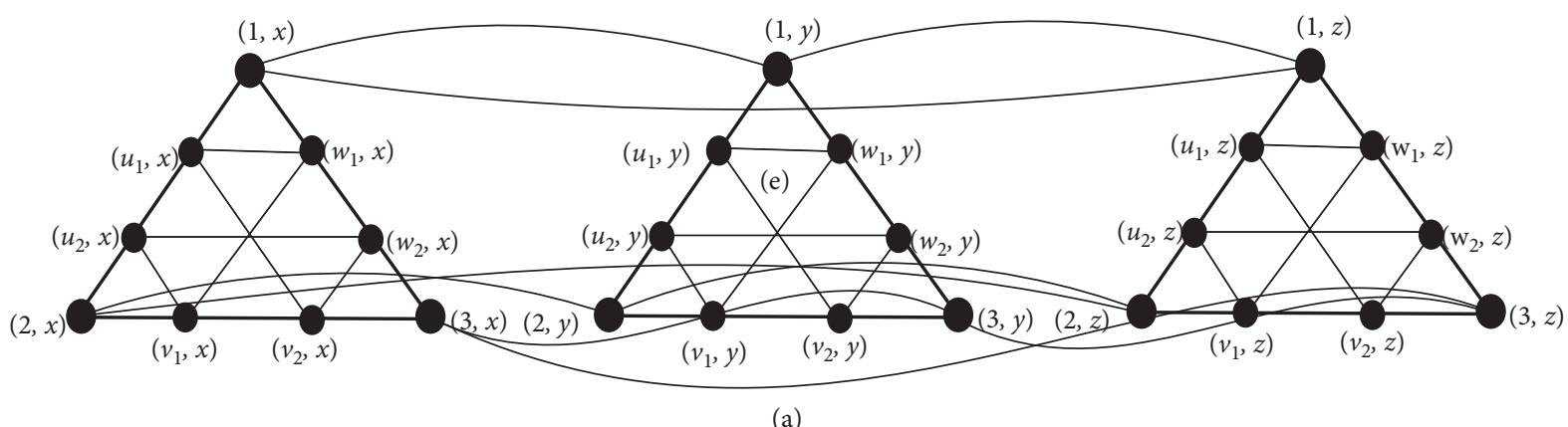

FIgUre 3: Continued. 


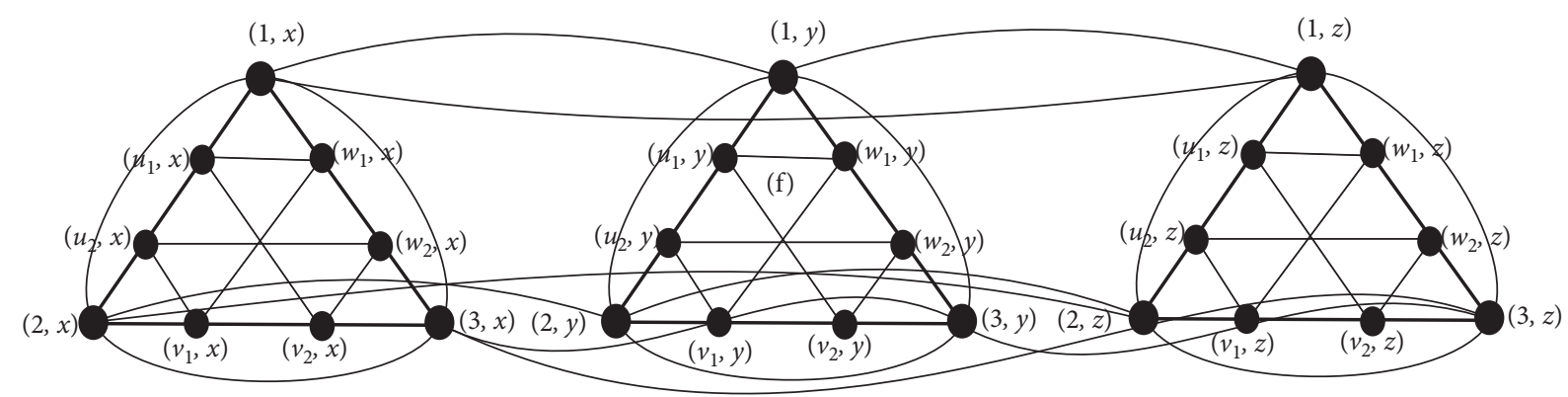

(b)

Figure 3: (a) $H_{1+Q_{2}} H_{2}$ and (b) $\Gamma_{1+T_{2}} \Gamma_{2}$.

$$
\begin{aligned}
\prod_{1}\left(\Gamma_{1}+s_{S_{k}} \Gamma_{2}\right)= & \prod_{\left(p_{1}, q_{1}\right)\left(p_{2}, q_{2}\right) \in E\left(\Gamma_{1}+s_{k} \Gamma_{2}\right)}\left[d\left(p_{1}, q_{1}\right)+d\left(p_{2}, q_{2}\right)\right] \\
= & \prod_{p \in V\left(\Gamma_{1}\right)} \prod_{q_{1} q_{2} \in E\left(\Gamma_{2}\right)}\left[d\left(p, q_{1}\right)+d\left(p, q_{2}\right)\right] \times \prod_{q \in V\left(\Gamma_{2}\right)} \prod_{\substack{p_{1} p_{2} \in E\left(s_{k}\left(\Gamma_{1}\right)\right) \\
p_{1} \in V\left(\Gamma_{1}\right), p_{2} \in V\left(s_{k}\left(\Gamma_{1}\right)-\Gamma_{1}\right)}}\left[d\left(p_{1}, q\right)+d\left(p_{2}, q\right)\right] \\
& \times \prod_{q \in V\left(\Gamma_{2}\right)} \prod_{p_{1} p_{2} \in E\left(s_{k}\left(\Gamma_{1}\right)\right)}\left[d\left(p_{1}, q\right)+d\left(p_{2}, q\right)\right] \\
= & \prod 1 \times \prod_{p_{1}, p_{2} \in V\left(s_{k}\left(\Gamma_{1}\right)-\Gamma_{1}\right)}^{2 \times \prod_{3}^{3} .}
\end{aligned}
$$

Now,

$$
\begin{aligned}
& \prod 1=\prod_{p \in V\left(\Gamma_{1}\right)} \prod_{q_{1} q_{2} \in E\left(\Gamma_{2}\right)}\left[d\left(p, q_{1}\right)+d\left(p, q_{2}\right)\right] \leq \frac{1}{\mu_{1} \omega_{2}}\left[\sum_{p \in V\left(\Gamma_{1}\right)} \sum_{q_{1} q_{2} \in E\left(\Gamma_{2}\right)}\left[d\left(p, q_{1}\right)+d\left(p, q_{2}\right)\right]\right]^{\mu_{1} \omega_{2}} \\
& =\frac{1}{\mu_{1} \omega_{2}}\left[\sum_{p \in V\left(\Gamma_{1}\right)} \sum_{q_{1} q_{2} \in E\left(\Gamma_{2}\right)}\left[2 d_{\Gamma_{1}}(p)+d_{\Gamma_{2}}\left(q_{1}\right)+d_{\Gamma_{2}}\left(q_{2}\right)\right]\right]^{\mu_{1} \omega_{2}}=\frac{1}{\mu_{1} \omega_{2}}\left[4 \omega_{1} \omega_{2}+\mu_{1} M_{1}\left(\Gamma_{2}\right)\right]^{\mu_{1} \omega_{2}}, \\
& \prod 2=\prod_{q \in V\left(\Gamma_{2}\right)} \prod_{p_{1} p_{2} \in E\left(S_{k}\left(\Gamma_{1}\right)\right)}\left[d\left(p_{1}, q\right)+d\left(p_{2}, q\right)\right] \leq \frac{1}{2 \omega_{1} \mu_{2}}\left[\sum_{q \in V\left(\Gamma_{2}\right)} \sum_{\substack{p_{1} p_{2} \in E\left(S_{k}\left(\Gamma_{1}\right)\right) \\
p_{1} \in V\left(\Gamma_{1}\right), p_{2} \in V\left(S_{k}\left(\Gamma_{1}\right)\right)-\mu\left(\Gamma_{1}\right)}}\left[d\left(p_{1}, q\right)+d\left(p_{2}, q\right)\right]\right]^{2 \omega_{1} \mu_{2}} \\
& =\frac{1}{2 \omega_{1} \mu_{2}}\left[\sum_{q \in V\left(\Gamma_{2}\right)} \sum_{\substack{p_{1} p_{2} \in E\left(S_{k}\left(\Gamma_{1}\right)\right) \\
p_{1} \in V\left(\Gamma_{1}\right), p_{2} \in V\left(S_{k}\left(\Gamma_{1}\right)\right)-\mu\left(\Gamma_{1}\right)}}\left[d_{S_{k}\left(\Gamma_{1}\right)}\left(p_{1}\right)+d_{\Gamma_{2}}(q)+d_{S_{k}\left(\Gamma_{1}\right)}\left(p_{2}\right)\right]\right]^{2 \omega_{1} \mu_{2}} \\
& =\frac{1}{2 \omega_{1} \mu_{2}}\left[4 \omega_{1} \omega_{2}+\mu_{2} M_{1}\left(S_{1}\left(\Gamma_{1}\right)\right)\right]^{2 \omega_{1} \mu_{2}} \text {, }
\end{aligned}
$$




$$
\begin{aligned}
& \prod^{3}=\prod_{q \in V\left(\Gamma_{2}\right)} \prod_{p_{1} p_{2} \in E\left(s_{k}\left(\Gamma_{1}\right)\right)}\left[d\left(p_{1}, q\right)+d\left(p_{2}, q\right)\right] \\
& p_{1}, p_{2} \in V\left(S_{k}\left(\Gamma_{1}\right)-\Gamma_{1}\right) \\
& \leq \frac{1}{\omega_{1} \mu_{2}(k-1)}\left[(k-1) \sum_{q \in V\left(\Gamma_{2}\right)} \sum_{\substack{p_{1} p_{2} \in E\left(S_{k}\left(\Gamma_{1}\right)\right) \\
p_{1}, p_{2} \in V\left(S_{k}\left(\Gamma_{1}\right)\right)-\mu\left(\Gamma_{1}\right)}}\left[d\left(p_{1}, q\right)+d\left(p_{2}, q\right)\right]\right]^{\omega_{1} \mu_{2}(k-1)} \\
& =\frac{1}{\omega_{1} \mu_{2}(k-1)}\left[(k-1) \sum_{q \in V\left(\Gamma_{2}\right)} \sum_{\substack{p_{1} p_{2} \in E\left(S_{k}\left(\Gamma_{1}\right)\right) \\
p_{1}, p_{2} \in V\left(S_{k}\left(\Gamma_{1}\right)\right)-\mu\left(\Gamma_{1}\right)}}[2+2]\right]^{\omega_{1} \mu_{2}(k-1)}=\frac{1}{\omega_{1} \mu_{2}(k-1)}\left[(k-1)\left|\omega\left(\Gamma_{1}\right)\right| \sum_{q \in V\left(\Gamma_{2}\right)}(4)\right]^{\omega_{1} \mu_{2}(k-1)} \\
& =\frac{1}{\omega_{1} \mu_{2}(k-1)}\left[4(k-1) \mu_{2} \omega_{1}\right]^{\omega_{1} \mu_{2}(k-1)} \text {. }
\end{aligned}
$$

Hence,

$$
\begin{aligned}
& \prod_{1}\left(\Gamma_{1}+{ }_{S_{k}} \Gamma_{2}\right) \leq \frac{1}{\mu_{1} \omega_{2}}\left[4 \omega_{1} \omega_{2}+\mu_{1} M_{1}\left(\Gamma_{2}\right)\right]^{\mu_{1} \omega_{2}} \times \frac{1}{2 \omega_{1} \mu_{2}}\left[4 \omega_{1} \omega_{2}+\mu_{2} M_{1}\left(S_{1}\left(\Gamma_{1}\right)\right)\right]^{2 \omega_{1} \mu_{2}} \\
& \times \frac{1}{\omega_{1} \mu_{2}(k-1)}\left[4(k-1) \mu_{2} \omega_{1}\right]^{\omega_{1} \mu_{2}(k-1)}, \\
& \text { (b) } \prod_{2}\left(\Gamma_{1}+{ }_{s_{k}} \Gamma_{2}\right)=\prod_{\left(p_{1}, q_{1}\right)}\left[d\left(p_{1}, q_{1}\right) d\left(p_{2}, q_{2}\right)\right] \\
& =\prod_{p \in V\left(\Gamma_{1}\right)} \prod_{q_{1} q_{2} \in E\left(\Gamma_{2}\right)}\left[d\left(p, q_{1}\right) d\left(p, q_{2}\right)\right] \\
& \times \prod_{q \in V\left(\Gamma_{2}\right)} \prod_{\substack{p_{1} p_{2} \in E\left(S_{k}\left(\Gamma_{1}\right)\right) \\
p_{1}, p_{2} \in V\left(S_{k}\left(\Gamma_{1}\right)\right)-\mu\left(\Gamma_{1}\right)}}\left[d\left(p_{1}, q\right) d\left(p_{2}, q\right)\right] 4 \\
& \times \prod_{q \in V\left(\Gamma_{2}\right)} \prod_{\substack{p_{1} p_{2} \in E\left(S_{k}\left(\Gamma_{1}\right)\right) \\
p_{1} \in V\left(\Gamma_{1}\right), p_{2} \in V\left(S_{k}\left(\Gamma_{1}\right)\right)-\mu\left(\Gamma_{1}\right)}}\left[d\left(p_{1}, q\right) d\left(p_{2}, q\right)\right] \\
& =\prod 1 \times \prod 2 \times \prod 3 .
\end{aligned}
$$


Now,

$$
\begin{aligned}
& \prod 1=\prod_{p \in V\left(\Gamma_{1}\right)} \prod_{q_{1} q_{2} \in E\left(\Gamma_{2}\right)}\left[d\left(p, q_{1}\right) d\left(p, q_{2}\right)\right] \leq \frac{1}{\mu_{1} \omega_{2}}\left[\sum_{p \in V\left(\Gamma_{1}\right)} \sum_{q_{1} q_{2} \in E\left(\Gamma_{2}\right)}\left[d\left(p, q_{1}\right) d\left(p, q_{2}\right)\right]\right]^{\mu_{1} \omega_{2}} \\
& =\frac{1}{\mu_{1} \omega_{2}}\left[\sum_{p \in V\left(\Gamma_{1}\right)} \sum_{q_{1} q_{2} \in E\left(\Gamma_{2}\right)}\left[d_{\Gamma_{1}}(p)+d_{\Gamma_{2}}\left(q_{1}\right)\right]\left[d_{\Gamma_{1}}(p)+d_{\Gamma_{2}}\left(q_{2}\right)\right]\right]^{\mu_{1} \omega_{2}} \\
& =\frac{1}{\mu_{1} \omega_{2}}\left[\omega_{2} M_{1}\left(\Gamma_{1}\right)+2 \omega_{1} M_{1}\left(\Gamma_{2}\right)+\mu_{1} M_{2}\left(\Gamma_{2}\right)\right]^{\mu_{1} \omega_{2}} \\
& \prod 2=\prod_{q \in V\left(\Gamma_{2}\right)} \prod_{\substack{p_{1} p_{2} \in E\left(S_{k}\left(\Gamma_{1}\right)\right) \\
p_{1} \in V\left(\Gamma_{1}\right), p_{2} \in V\left(S_{k}\left(\Gamma_{1}\right)\right)-\mu\left(\Gamma_{1}\right)}}\left[d\left(p_{1}, q\right) d\left(p_{2}, q\right)\right] \\
& \leq \frac{1}{2 \mu_{2} \omega_{1}}\left[\sum_{\substack { q \in V\left(\Gamma_{2}\right) \\
\begin{subarray}{c}{p_{1} p_{2} \in E\left(S_{k}(\Gamma)\right) \\
p_{1} \in V\left(\Gamma_{1}\right), p_{2} \in V\left(S_{k}\left(\Gamma_{1}\right)\right)-\mu\left(\Gamma_{1}\right){ q \in V ( \Gamma _ { 2 } ) \\
\begin{subarray} { c } { p _ { 1 } p _ { 2 } \in E ( S _ { k } ( \Gamma ) ) \\
p _ { 1 } \in V ( \Gamma _ { 1 } ) , p _ { 2 } \in V ( S _ { k } ( \Gamma _ { 1 } ) ) - \mu ( \Gamma _ { 1 } ) } }\end{subarray}}\left[d\left(p_{1}, q\right) d\left(p_{2}, q\right)\right]\right]^{2 \mu_{2} \omega_{1}} \\
& =\frac{1}{2 \mu_{2} \omega_{1}}\left[\sum_{\substack { q \in V\left(\Gamma_{2}\right) \\
\begin{subarray}{c}{p_{1} p_{2} \in E\left(S_{k}\left(\Gamma_{1}\right)\right) \\
p_{1} \in V\left(\Gamma_{1}\right), p_{2} \in V\left(S_{k}\left(\Gamma_{1}\right)\right)-\mu\left(\Gamma_{1}\right){ q \in V ( \Gamma _ { 2 } ) \\
\begin{subarray} { c } { p _ { 1 } p _ { 2 } \in E ( S _ { k } ( \Gamma _ { 1 } ) ) \\
p _ { 1 } \in V ( \Gamma _ { 1 } ) , p _ { 2 } \in V ( S _ { k } ( \Gamma _ { 1 } ) ) - \mu ( \Gamma _ { 1 } ) } }\end{subarray}}\left[d_{S_{k}\left(\Gamma_{1}\right)}\left(p_{1}\right)+d_{\Gamma_{2}}(q)\right] d_{S_{k}\left(\Gamma_{1}\right)}\left(p_{2}\right)\right]^{2 \mu_{2} \omega_{1}}, \\
& \prod 3=\sum_{q \in V\left(\Gamma_{2}\right)} \sum_{\substack{p_{1} p_{2} \in E\left(S_{k}\left(\Gamma_{1}\right)\right) \\
p_{1} \in V\left(\Gamma_{1}\right), p_{2} \in V\left(S_{k}\left(\Gamma_{1}\right)\right)-\mu\left(\Gamma_{1}\right)}}\left[d\left(p_{1}, q\right) d\left(p_{2}, q\right)\right] \\
& \leq \frac{1}{(k-1) \mu_{2} \omega_{1}}\left[\prod_{q \in V\left(\Gamma_{2}\right)} \prod_{\substack{p_{1} p_{2} \in E\left(S_{k}\left(\Gamma_{1}\right)\right) \\
p_{1}, p_{2} \in V\left(S_{k}\left(\Gamma_{1}\right)\right)-\mu\left(\Gamma_{1}\right)}}\left[d\left(p_{1}, q\right) d\left(p_{2}, q\right)\right]\right]^{(k-1) \mu_{2} \omega_{1}} \\
& =\frac{1}{(k-1) \mu_{2} \omega_{1}}\left[\sum_{q \in V\left(\Gamma_{2}\right)} \sum_{\substack{p_{1} p_{2} \in E\left(S_{k}\left(\Gamma_{1}\right)\right) \\
p_{1}, p_{2} \in V\left(S_{k}\left(\Gamma_{1}\right)\right)-\mu\left(\Gamma_{1}\right)}}[4]\right]^{(k-1) \mu_{2} \omega_{1}}=\frac{1}{(k-1) \mu_{2} \omega_{1}}\left[4(k-1) \mu_{2} \omega_{1}\right]^{(k-1) \mu_{2} \omega_{1}} .
\end{aligned}
$$


Consequently,

$$
\begin{aligned}
\prod_{2}\left(\Gamma_{1}+{ }_{S_{k}} \Gamma_{2}\right) \leq & \frac{1}{\mu_{1} \omega_{2}}\left[\omega_{2} M_{1}\left(\Gamma_{1}\right)+2 \omega_{1} M_{1}\left(\Gamma_{2}\right)+\mu_{1} M_{2}\left(\Gamma_{2}\right)\right]^{\mu_{1} \omega_{2}} \\
& \times \frac{1}{2 \mu_{2} \omega_{1}}\left[\mu_{2} M_{2}\left(S_{1}\left(\Gamma_{1}\right)\right)+8 \omega_{1} \omega_{2}\right]^{2 \mu_{2} \omega_{1}} \\
& \times \frac{1}{(k-1) \mu_{2} \omega_{1}}\left[4(k-1) \mu_{2} \omega_{1}\right]^{(k-1) \mu_{2} \omega_{1}} .
\end{aligned}
$$

Theorem 2. Let $\Gamma_{1}, \Gamma_{2}$ be two graphs with $\left|V\left(\Gamma_{1}\right)\right|=\mu_{1}$, $\left|V\left(\Gamma_{2}\right)\right|=\mu_{2},\left|E\left(\Gamma_{1}\right)\right|=\omega_{1}$, and $\left|E\left(\Gamma_{2}\right)\right|=\omega_{2}$. For $\mu_{1}, \mu_{2} \geq 4$ and $k \geq 1$, we have

$$
\begin{aligned}
\prod_{1}\left(\Gamma_{1}+{ }_{R_{k}} \Gamma_{2}\right) \leq & \frac{1}{\mu_{1} \omega_{2}}\left[\mu_{1} M_{1}\left(\Gamma_{2}\right)+8 \omega_{1} \omega_{2}\right]^{\mu_{1} \omega_{2}} \times \frac{1}{\omega_{1} \mu_{2}}\left[4 \omega_{1} \omega_{2}+2 \mu_{2} M_{1}\left(\Gamma_{1}\right)\right]^{\omega_{1} \mu_{2}} \\
& \times \frac{1}{2 \omega_{1} \mu_{2}}\left[4 \omega_{1} \omega_{2}+\mu_{2}\left(M_{1} R_{1}\left(\Gamma_{1}\right)-2 M_{1}\left(\Gamma_{1}\right)\right)\right]^{2 \omega_{1} \mu_{2}} \times \frac{1}{\omega_{1} \mu_{2}(k-1)}\left[4(k-1) \mu_{2} \omega_{1}\right]^{\omega_{1} \mu_{2}(k-1)}, \\
\prod_{2}\left(\Gamma_{1}+{ }_{R_{k}} \Gamma_{2}\right) \leq & \frac{1}{\omega_{2} \mu_{1}}\left[4 \omega_{2} M_{1}\left(\Gamma_{1}\right)+4 \omega_{1} M_{1}\left(\Gamma_{2}\right)+\mu_{1} M_{2}\left(\Gamma_{2}\right)\right]^{\omega_{2} \mu_{1}} \\
& \times \frac{1}{\omega_{1} \mu_{2}}\left[4 \mu_{2} M_{2}\left(\Gamma_{1}\right)+4 \omega_{2} M_{1}\left(\Gamma_{1}\right)+\omega_{1} M_{1}\left(\Gamma_{2}\right)\right]^{\omega_{1} \mu_{2}} \\
& \times \frac{1}{2 \omega_{1} \mu_{2}}\left[8 \omega_{1} \omega_{2}+\mu_{2}\left(M_{2}\left(R_{1}\left(\Gamma_{1}\right)\right)-4 M_{2}\left(\Gamma_{1}\right)\right)\right]^{2 \omega_{1} \mu_{2}} \times \frac{1}{(k-1) \omega_{1} \mu_{2}}\left[4 \mu_{2} \omega_{1}(k-1)\right]^{(k-1) \omega_{1} \mu_{2}} .
\end{aligned}
$$

Proof

(a) Consider $d(p, q)=d_{\left(\Gamma_{1}+{ }_{R_{k}} \Gamma_{2}\right)}(p, q)$ as a degree of a node $(p, q)$ in $\Gamma_{1}+{ }_{R_{k}} \Gamma_{2}$ :

$$
\begin{aligned}
\prod_{1}\left(\Gamma_{1}+{ }_{R_{k}} \Gamma_{2}\right)= & \prod_{\left(p_{1}, q_{1}\right)}\left[d\left(p_{2}, q_{2}\right) \in E\left(\Gamma_{1}+R_{k} \Gamma_{2}\right)\right. \\
= & \prod_{p \in V\left(\Gamma_{1}\right)} \prod_{q_{1} q_{2} \in E\left(\Gamma_{2}\right)}\left[d\left(p, q_{1}\right)+d\left(p, q_{2}\right)\right] \\
& \times \prod_{q \in V\left(\Gamma_{2}\right)} \prod_{p_{1} p_{2} \in E\left(R_{k}\left(\Gamma_{1}\right)\right)}\left[d\left(p_{1}, q\right)+d\left(p_{2}, q\right)\right] \\
= & \prod 1 \times \prod 2 .
\end{aligned}
$$


Now,

$$
\begin{aligned}
& \prod 1=\prod_{p \in V\left(\Gamma_{1}\right)} \prod_{q_{1} q_{2} \in E\left(\Gamma_{2}\right)}\left[d\left(p, q_{1}\right)+d\left(p, q_{2}\right)\right] \leq \frac{1}{\mu_{1} \omega_{2}}\left[\sum_{p \in V\left(\Gamma_{1}\right)} \sum_{q_{1} q_{2} \in E\left(\Gamma_{2}\right)}\left[d\left(p, q_{1}\right)+d\left(p, q_{2}\right)\right]\right]^{\mu_{1} \omega_{2}} \\
& =\frac{1}{\mu_{1} \omega_{2}}\left[\sum_{p \in V\left(\Gamma_{1}\right)} \sum_{q_{1} q_{2} \in E\left(\Gamma_{2}\right)}\left[2 d_{R_{k}\left(\Gamma_{1}\right)}(p)+d_{\Gamma_{2}}\left(q_{1}\right)+d_{\Gamma_{2}}\left(q_{2}\right)\right]\right]^{\mu_{1} \omega_{2}} \\
& =\frac{1}{\mu_{1} \omega_{2}}\left[\sum_{p \in V\left(\Gamma_{1}\right)} \sum_{q_{1} q_{2} \in E\left(\Gamma_{2}\right)}\left[4 d_{\Gamma_{1}}(p)+d_{\Gamma_{2}}\left(q_{1}\right)+d_{\Gamma_{2}}\left(q_{2}\right)\right]\right]^{\mu_{1} \omega_{2}}=\frac{1}{\mu_{1} \omega_{2}}\left[\mu_{1} M_{1}\left(\Gamma_{2}\right)+8 \omega_{1} \omega_{2}\right]^{\mu_{1} \omega_{2}}, \\
& \prod 2=\prod_{q \in V\left(\Gamma_{2}\right)} \prod_{p_{1} p_{2} \in E\left(R_{k}\left(\Gamma_{1}\right)\right)}\left[d\left(p_{1}, q\right)+d\left(p_{2}, q\right)\right]=\prod_{q \in V\left(\Gamma_{2}\right)} \prod_{p_{1} p_{2} \in E\left(R_{k}\left(\Gamma_{1}\right)\right)}\left[d\left(p_{1}, q\right)+d\left(p_{2}, q\right)\right] \\
& p_{1}, p_{2} \in V\left(\Gamma_{1}\right) \\
& \times \prod_{q \in V\left(\Gamma_{2}\right)} \prod_{\substack{p_{1} p_{2} \in E\left(R_{k}\left(\Gamma_{1}\right)\right) \\
p_{1} \in V\left(\Gamma_{1}\right)}}\left[d\left(p_{1}, q\right)+d\left(p_{2}, q\right)\right] \times \prod_{\substack { q \in V\left(\Gamma_{2}\right) \\
\begin{subarray}{c}{p_{1}, p_{2} \in V\left(R_{k}\left(\Gamma_{1}\right)\right)-\mu\left(\Gamma_{1}\right){ q \in V ( \Gamma _ { 2 } ) \\
\begin{subarray} { c } { p _ { 1 } , p _ { 2 } \in V ( R _ { k } ( \Gamma _ { 1 } ) ) - \mu ( \Gamma _ { 1 } ) } }\end{subarray}}\left[d\left(p_{1}, q\right)+d\left(p_{2}, q\right)\right] \\
& =\prod^{\prime} 2 \times \prod^{\prime \prime} 2 \times \prod^{\prime \prime \prime} 2 .
\end{aligned}
$$

Now, for $p_{1} p_{2} \in V\left(\Gamma_{1}\right)$, then $p_{1} p_{2} \in E\left(R_{k}\left(\Gamma_{1}\right)\right)$ if and only if $p_{1} p_{2} \in E\left(\Gamma_{1}\right)$; for $p_{1} \in V\left(\Gamma_{1}\right)$, we get $d_{R_{k}\left(\Gamma_{1}\right)}\left(p_{1}\right)=$

$2 d_{\Gamma_{1}}\left(p_{1}\right)$ and for $p_{2} \in V\left(R_{k}\left(\Gamma_{1}\right)\right)-\mu\left(\Gamma_{1}\right)$, we have $d_{R_{k}\left(\Gamma_{1}\right)}\left(p_{2}\right)=2$. Now,

$$
\begin{aligned}
& \prod^{\prime} 2=\prod_{q \in V\left(\Gamma_{2}\right)} \prod_{\substack{p_{1} p_{2} \in E\left(R_{k}\left(\Gamma_{1}\right)\right) \\
p_{1}, p_{2} \in V\left(\Gamma_{1}\right)}}\left[d\left(p_{1}, q\right)+d\left(p_{2}, q\right)\right] \leq \frac{1}{\omega_{1} \mu_{2}}\left[\sum_{\substack{q \in V\left(\Gamma_{2}\right) \\
p_{1} p_{2} \in E\left(R_{k}\left(\Gamma_{1}\right)\right) \\
p_{1} p_{2} \in V\left(\Gamma_{1}\right)}}\left[d\left(p_{1}, q\right)+d\left(p_{2}, q\right)\right]\right]^{\omega_{1} \mu_{2}} \\
& =\frac{1}{\omega_{1} \mu_{2}}\left[\sum_{q \in V\left(\Gamma_{2}\right)} \sum_{p_{1} p_{2} \in E\left(\Gamma_{1}\right)}\left[2 d_{\Gamma_{2}}(q)\right]+\sum_{q \in V\left(\Gamma_{2}\right)} \sum_{p_{1} p_{2} \in E\left(\Gamma_{1}\right)}\left[d_{R_{k}\left(\Gamma_{1}\right)}\left(p_{1}\right)+d_{R_{k}\left(\Gamma_{1}\right)}\left(p_{2}\right)\right]\right]^{\omega_{1} \mu_{2}}=\frac{1}{\omega_{1} \mu_{2}}\left[4 \omega_{1} \omega_{2}+2 \mu_{2} M_{1}\left(\Gamma_{1}\right)\right]^{\omega_{1} \mu_{2}} \text {, } \\
& \prod^{\prime \prime} 2=\prod_{\substack{q \in V\left(\Gamma_{2}\right) \\
p_{1} p_{2} \in E\left(R_{k}\left(\Gamma_{1}\right)\right) \\
p_{1} \in V\left(\Gamma_{1}\right)}}\left[d\left(p_{1}, q\right)+d\left(p_{2}, q\right)\right] \leq \frac{1}{2 \omega_{1} \mu_{2}}\left[\sum_{\substack{q \in V\left(\Gamma_{2}\right) \\
p_{1} p_{2} \in E\left(R_{k}\left(\Gamma_{1}\right)\right) \\
p_{1} \in V\left(\Gamma_{1}\right)}}\left[d\left(p_{1}, q\right)+d\left(p_{2}, q\right)\right]\right]^{2 \omega_{1} \mu_{2}} \\
& =\frac{1}{2 \omega_{1} \mu_{2}}\left[\sum_{\substack{q \in V\left(\Gamma_{2}\right) \\
p_{1} p_{2} \in E\left(R_{k}\left(\Gamma_{1}\right)\right) \\
p_{1} \in V\left(\Gamma_{1}\right)}}\left[d_{R_{k}\left(\Gamma_{1}\right)}\left(p_{1}\right)+d_{\Gamma_{2}}(q)+d_{R_{k}\left(\Gamma_{1}\right)}\left(p_{2}\right)\right]\right]^{2 \omega_{1} \mu_{2}}=\frac{1}{2 \omega_{1} \mu_{2}}\left[4 \omega_{1} \omega_{2}+\mu_{2}\left(M_{1} R_{1}\left(\Gamma_{1}\right)-2 M_{1}\left(\Gamma_{1}\right)\right)\right]^{2 \omega_{1} \mu_{2}} \\
& \prod^{\prime \prime \prime} 2=\prod_{q \in V\left(\Gamma_{2}\right)} \prod_{\substack{p_{1} p_{2} \in E\left(R_{k}\left(\Gamma_{1}\right)\right) \\
p_{1}, p_{2} \in V\left(R_{k}\left(\Gamma_{1}\right)\right)-\mu\left(\Gamma_{1}\right)}}\left[d\left(p_{1}, q\right)+d\left(p_{2}, q\right)\right] \\
& \leq \frac{1}{\omega_{1} \mu_{2}(k-1)}\left[\sum_{q \in V\left(\Gamma_{2}\right)} \sum_{\substack{p_{1} p_{2} \in E\left(R_{k}\left(\Gamma_{1}\right)\right) \\
p_{1}, p_{2} \in V\left(R_{k}\left(\Gamma_{1}\right)\right)-\mu\left(\Gamma_{1}\right)}}\left[d\left(p_{1}, q\right)+d\left(p_{2}, q\right)\right]\right]^{\omega_{1} \mu_{2}(k-1)}=\frac{1}{\omega_{1} \mu_{2}(k-1)}\left[4(k-1) \mu_{2} \omega_{1}\right]^{\omega_{1} \mu_{2}(k-1)} .
\end{aligned}
$$


Hence,

$$
\begin{aligned}
\prod_{1}\left(\Gamma_{1}+{ }_{R_{k}} \Gamma_{2}\right) \leq & \frac{1}{\mu_{1} \omega_{2}}\left[\mu_{1} M_{1}\left(\Gamma_{2}\right)+8 \omega_{1} \omega_{2}\right]^{\mu_{1} \omega_{2}} \times \frac{1}{\omega_{1} \mu_{2}}\left[4 \omega_{1} \omega_{2}+2 \mu_{2} M_{1}\left(\Gamma_{1}\right)\right]^{\omega_{1} \mu_{2}} \\
& \times \frac{1}{2 \omega_{1} \mu_{2}}\left[4 \omega_{1} \omega_{2}+\mu_{2}\left(M_{1} R_{1}\left(\Gamma_{1}\right)-2 M_{1}\left(\Gamma_{1}\right)\right)\right]^{2 \omega_{1} \mu_{2}} \times \frac{1}{\omega_{1} \mu_{2}(k-1)}\left[4(k-1) \mu_{2} \omega_{1}\right]^{\omega_{1} \mu_{2}(k-1)}, \\
\text { (b) } \prod_{2}\left(\Gamma_{1}+{ }_{R_{k}} \Gamma_{2}\right)= & \prod_{\left(p_{1}, q_{1}\right)\left(p_{2}, q_{2}\right) \cdot \in E\left(\Gamma_{1}++_{R_{k}} \Gamma_{2}\right)}\left[d\left(p_{1}, q_{1}\right) d\left(p_{2}, q_{2}\right)\right] \\
= & \prod_{p \in V\left(\Gamma_{1}\right) q_{1} q_{2} \in E\left(\Gamma_{2}\right)}\left[d\left(p, q_{1}\right) d\left(p, q_{2}\right)\right] \\
& \times \prod_{q \in V\left(\Gamma_{2}\right) p_{1} p_{2} \in E\left(R_{k}\left(\Gamma_{1}\right)\right)}\left[d\left(p_{1}, q\right) d\left(p_{2}, q\right)\right] \\
= & \prod 1 \times \prod_{1}^{2 .}
\end{aligned}
$$

Now,

$$
\begin{aligned}
& \prod 1=\prod_{p \in V\left(\Gamma_{1}\right)} \prod_{q_{1} q_{2} \in E\left(\Gamma_{2}\right)}\left[d\left(p, q_{1}\right) d\left(p, q_{2}\right)\right] \leq \frac{1}{\omega_{2} \mu_{1}}\left[\sum_{p \in V\left(\Gamma_{1}\right)} \sum_{q_{1} q_{2} \in E\left(\Gamma_{2}\right)}\left[d\left(p, q_{1}\right) d\left(p, q_{2}\right)\right]\right]^{\omega_{2} \mu_{1}} \\
& =\frac{1}{\omega_{2} \mu_{1}}\left[\sum_{p \in V\left(\Gamma_{1}\right)} \sum_{q_{1} q_{2} \in E\left(\Gamma_{2}\right)}\left[d_{R_{k}\left(\Gamma_{1}\right)}(p)+d_{\Gamma_{2}}\left(q_{1}\right)\right]\left[d_{R_{k}\left(\Gamma_{1}\right)}(p)+d_{\Gamma_{2}}\left(q_{2}\right)\right]\right]^{\omega_{2} \mu_{1}} \\
& =\frac{1}{\omega_{2} \mu_{1}}\left[4 \omega_{2} M_{1}\left(\Gamma_{1}\right)+4 \omega_{1} M_{1}\left(\Gamma_{2}\right)+\mu_{1} M_{2}\left(\Gamma_{2}\right)\right]^{\omega_{2} \mu_{1}}, \\
& \prod 2=\prod_{q \in V\left(\Gamma_{2}\right)} \prod_{p_{1} p_{2} \in E\left(R_{k}\left(\Gamma_{1}\right)\right)}\left[d\left(p_{1}, q\right) d\left(p_{2}, q\right)\right] \\
& =\prod_{\substack{q \in V\left(\Gamma_{2}\right) \\
p_{1} p_{2} \in E\left(R_{k}\left(\Gamma_{1}\right)\right) \\
p_{1}, p_{2} \in V\left(\Gamma_{1}\right)}}\left[d\left(p_{1}, q\right) d\left(p_{2}, q\right)\right] \times \prod_{\substack { q \in V\left(\Gamma_{2}\right) \\
\begin{subarray}{c}{p_{1} p_{2} \in E\left(R_{k}\left(\Gamma_{1}\right)\right) \\
p_{1} \in V\left(\Gamma_{1}\right) \\
p_{2} \in V\left(R_{k}\left(\Gamma_{1}\right)\right)-\mu\left(\Gamma_{2}\right){ q \in V ( \Gamma _ { 2 } ) \\
\begin{subarray} { c } { p _ { 1 } p _ { 2 } \in E ( R _ { k } ( \Gamma _ { 1 } ) ) \\
p _ { 1 } \in V ( \Gamma _ { 1 } ) \\
p _ { 2 } \in V ( R _ { k } ( \Gamma _ { 1 } ) ) - \mu ( \Gamma _ { 2 } ) } }\end{subarray}}\left[d\left(p_{1}, q\right) d\left(p_{2}, q\right)\right] \\
& \times \prod_{q \in V\left(\Gamma_{2}\right)} \prod_{\substack{p_{1} p_{2} \in E\left(R_{k}\left(\Gamma_{1}\right)\right) \\
p_{1}, p_{2} \in V\left(R_{k}\left(\Gamma_{1}\right)\right)-\mu\left(\Gamma_{1}\right)}}\left[d\left(p_{1}, q\right) d\left(p_{2}, q\right)\right] \\
& =\prod^{\prime} 2 \times \prod^{\prime \prime} 2 \times \prod^{\prime \prime \prime} 2 .
\end{aligned}
$$


Now, we consider

$$
\begin{aligned}
& \prod^{\prime} 2=\prod_{q \in V\left(\Gamma_{2}\right)} \prod_{p_{1} p_{2} \in E\left(R_{k}\left(\Gamma_{1}\right)\right)}\left[d\left(p_{1}, q\right) d\left(p_{2}, q\right)\right]=\prod_{q \in V\left(\Gamma_{2}\right)} \prod_{p_{1} p_{2} \in E\left(\Gamma_{1}\right)}\left[d\left(p_{1}, q\right) d\left(p_{2}, q\right)\right] \\
& \leq \frac{1}{\omega_{1} \mu_{2}}\left[\sum_{q \in V\left(\Gamma_{2}\right)} \sum_{p_{1} p_{2} \in E\left(\Gamma_{1}\right)}\left[d\left(p_{1}, q\right) d\left(p_{2}, q\right)\right]\right]^{\omega_{1} \mu_{2}} \\
& =\frac{1}{\omega_{1} \mu_{2}}\left[\sum_{q \in V\left(\Gamma_{2}\right)} \sum_{p_{1} p_{2} \in E\left(\Gamma_{1}\right)}\left[d_{R_{k}\left(\Gamma_{1}\right)}\left(p_{1}\right)+d_{\Gamma_{2}}(q)\right]\left[d_{R_{k}\left(\Gamma_{1}\right)}\left(p_{2}\right)+d_{\Gamma_{2}}(q)\right]\right]^{\omega_{1} \mu_{2}} \\
& =\frac{1}{\omega_{1} \mu_{2}}\left[4 \mu_{2} M_{2}\left(\Gamma_{1}\right)+4 \omega_{2} M_{1}\left(\Gamma_{1}\right)+\omega_{1} M_{1}\left(\Gamma_{2}\right)\right]^{\omega_{1} \mu_{2}} \\
& \prod^{\prime \prime} 2=\prod_{q \in V\left(\Gamma_{2}\right)} \prod_{\substack{p_{1} p_{2} \in E\left(R_{k}\left(\Gamma_{1}\right)\right) \\
p_{1} \in V\left(\Gamma_{1}\right)}}\left[d\left(p_{1}, q\right) d\left(p_{2}, q\right)\right] \\
& \leq \frac{1}{2 \omega_{1} \mu_{2}}\left[\sum_{\substack{q \in V\left(\Gamma_{2}\right) \\
p_{1} p_{2} \in E\left(R_{k}\left(\Gamma_{1}\right)\right) \\
p_{1} \in V\left(\Gamma_{1}\right)}}\left[d\left(p_{1}, q\right) d\left(p_{2}, q\right)\right]\right]^{2 \omega_{1} \mu_{2}}=\frac{1}{2 \omega_{1} \mu_{2}}\left[\sum_{\substack{q\left(\Gamma_{2}\right) \\
p_{1} p_{2} \in E\left(R_{k}\left(\Gamma_{1}\right)\right) \\
p_{1} \in V\left(\Gamma_{1}\right)}}\left[d_{R_{k}\left(\Gamma_{1}\right)}\left(p_{1}\right)+d_{\Gamma_{2}}(q)\right] \times 2\right]^{2 \omega_{1} \mu_{2}} \\
& =\frac{1}{2 \omega_{1} \mu_{2}}\left[8 \omega_{1} \omega_{2}+\mu_{2}\left(M_{2}\left(R_{1}\left(\Gamma_{1}\right)\right)-4 M_{2}\left(\Gamma_{1}\right)\right)\right]^{2 \omega_{1} \mu_{2}} \text {, } \\
& \prod^{\prime \prime \prime} 2=\prod_{q \in V\left(\Gamma_{2}\right)} \prod_{\substack{p_{1} p_{2} \in E\left(R_{k}\left(\Gamma_{1}\right)\right) \\
p_{1} p_{2} \in V\left(R_{k}\left(\Gamma_{1}\right)\right)-\mu\left(\Gamma_{1}\right)}}\left[d\left(p_{1}, q\right) d\left(p_{2}, q\right)\right] \\
& \leq \frac{1}{(k-1) \omega_{1} \mu_{2}}\left[(k-1) \sum_{\substack { q \in V\left(\Gamma_{2}\right) \\
\begin{subarray}{c}{p_{1} p_{2} \in E\left(R_{k}\left(\Gamma_{1}\right)\right) \\
p_{1} p_{2} \in V\left(R_{k}\left(\Gamma_{1}\right)\right)-\mu\left(\Gamma_{1}\right){ q \in V ( \Gamma _ { 2 } ) \\
\begin{subarray} { c } { p _ { 1 } p _ { 2 } \in E ( R _ { k } ( \Gamma _ { 1 } ) ) \\
p _ { 1 } p _ { 2 } \in V ( R _ { k } ( \Gamma _ { 1 } ) ) - \mu ( \Gamma _ { 1 } ) } }\end{subarray}}\left[d\left(p_{1}, q\right) d\left(p_{2}, q\right)\right]\right]^{(k-1) \omega_{1} \mu_{2}}=\frac{1}{(k-1) \omega_{1} \mu_{2}}\left[4 \mu_{2} \omega_{1}(k-1)\right]^{(k-1) \omega_{1} \mu_{2}}
\end{aligned}
$$

Therefore, we have

$$
\begin{aligned}
\prod_{2}\left(\Gamma_{1}+{ }_{R_{k}} \Gamma_{2}\right) \leq & \frac{1}{\omega_{2} \mu_{1}}\left[4 \omega_{2} M_{1}\left(\Gamma_{1}\right)+4 \omega_{1} M_{1}\left(\Gamma_{2}\right)+\mu_{1} M_{2}\left(\Gamma_{2}\right)\right]^{\omega_{2} \mu_{1}} \\
& \times \frac{1}{\omega_{1} \mu_{2}}\left[4 \mu_{2} M_{2}\left(\Gamma_{1}\right)+4 \omega_{2} M_{1}\left(\Gamma_{1}\right)+\omega_{1} M_{1}\left(\Gamma_{2}\right)\right]^{\omega_{1} \mu_{2}} \\
& \times \frac{1}{2 \omega_{1} \mu_{2}}\left[8 \omega_{1} \omega_{2}+\mu_{2}\left(M_{2}\left(R_{1}\left(\Gamma_{1}\right)\right)-4 M_{2}\left(\Gamma_{1}\right)\right)\right]^{2 \omega_{1} \mu_{2}} \times \frac{1}{(k-1) \omega_{1} \mu_{2}}\left[4 \mu_{2} \omega_{1}(k-1)\right]^{(k-1) \omega_{1} \mu_{2}}
\end{aligned}
$$


Theorem 3. Let $\Gamma_{1}, \Gamma_{2}$ be two graphs with $\left|V\left(\Gamma_{1}\right)\right|=\mu_{1}$, $\left|V\left(\Gamma_{2}\right)\right|=\mu_{2},\left|E\left(\Gamma_{1}\right)\right|=\omega_{1}$, and $\left|E\left(\Gamma_{2}\right)\right|=\omega_{2}$. For $\mu_{1}, \mu_{2} \geq 4$ and and $k \geq 1$, we have

$$
\begin{aligned}
& \prod_{1}\left(\Gamma_{1}+{ }_{Q_{k}} \Gamma_{2}\right) \leq \frac{1}{\mu_{1} \omega_{2}}\left[4 \omega_{1} \omega_{2}+\mu_{1} M_{1}\left(\Gamma_{2}\right)\right]^{\mu_{1} \omega_{2}} \times \frac{1}{2 \omega_{1} \mu_{2}}\left[4 \omega_{1} \omega_{2}+3 \mu_{2} M_{1}\left(\Gamma_{1}\right)\right]^{2 \omega_{1} \mu_{2}} \\
& \times \frac{1}{\omega_{1} \mu_{2}(k-1)}\left[2(k-1) \mu_{2} M_{1}\left(\Gamma_{1}\right)\right]^{\omega_{1} \mu_{2}(k-1)} \\
& \times \frac{1}{k \mu_{2}\left(2 \omega_{1}-\mu_{1}\right)}\left[(k) \mu_{2}\left[M_{3}\left(\Gamma_{1}\right)+2 M_{2}\left(\Gamma_{1}\right)-2 M_{1}\left(\Gamma_{1}\right)\right]\right]^{k \mu_{2}\left(2 \omega_{1}-\mu_{1}\right)}, \\
& \prod_{2}\left(\Gamma_{1}+{ }_{Q_{k}} \Gamma_{2}\right) \leq \frac{1}{\mu_{1} \omega_{2}}\left[\omega_{2} M_{1}\left(\Gamma_{1}\right)+2 \omega_{1} M_{1}\left(\Gamma_{2}\right)+\mu_{1} M_{2}\left(\Gamma_{2}\right)\right]^{\mu_{1} \omega_{2}} \\
& \times \frac{1}{2 \mu_{2} \omega_{1}}\left[4 \omega_{2} M_{1}\left(\Gamma_{1}\right)+\mu_{2}\left[M_{3}\left(\Gamma_{1}\right)+2 M_{2}\left(\Gamma_{1}\right)\right]\right]^{2 \mu_{2} \omega_{1}} \\
& \times \frac{1}{(k-1) \mu_{2} \omega_{1}}\left[(k-1) \mu_{2}\left[2 M_{2}\left(\Gamma_{1}\right)+M_{3}\left(\Gamma_{1}\right)\right]\right]^{(k-1) \mu_{2} \omega_{1}} \\
& \times \frac{1}{k \mu_{2}\left(2 \omega_{1}-\mu_{1}\right)}\left[(k) \mu_{2}\left[\frac{1}{2} M_{4}\left(\Gamma_{1}\right)-\frac{1}{2} M_{3}\left(\Gamma_{1}\right)\right]+\sum_{u v \in V\left(\Gamma_{1}\right)} r d_{\Gamma_{1}}(u) d_{\Gamma_{1}}(v)\right]^{k \mu_{2}\left(2 \omega_{1}-\mu_{1}\right)} \\
& +\frac{1}{k \mu_{2}\left(2 \omega_{1}-\mu_{1}\right)}\left[\sum_{v \in V\left(\Gamma_{1}\right)} d_{\Gamma_{1}}^{2}(v) \sum_{\substack{u \in V\left(\Gamma_{1}\right) \\
u v \in E\left(\Gamma_{1}\right)}} d_{\Gamma_{1}}(u)-2 M_{2}\left(\Gamma_{1}\right)\right]^{k \mu_{2}\left(2 \omega_{1}-\mu_{1}\right)} .
\end{aligned}
$$

Proof.

$$
\text { (a) } \begin{aligned}
\prod_{1}\left(\Gamma_{1}+{ }_{Q_{k}} \Gamma_{2}\right) & =\prod_{\left(p_{1}, q_{1}\right)\left(p_{2}, q_{2}\right) \in E\left(\Gamma_{1}+Q_{k} \Gamma_{2}\right)}\left[d\left(p_{1}, q_{1}\right)+d\left(p_{2}, q_{2}\right)\right] \\
& =\prod_{p \in V\left(\Gamma_{1}\right)} \prod_{q_{1} q_{2} \in E\left(\Gamma_{2}\right)}\left[d\left(p, q_{1}\right)+d\left(p, q_{2}\right)\right] \times \prod_{q \in V\left(\Gamma_{2}\right)} \prod_{p_{1} p_{2} \in E\left(Q_{k}\left(\Gamma_{2}\right)\right)}\left[d\left(p_{1}, q\right)+d\left(p_{2}, q\right)\right] \\
& =\prod 1 \times \prod^{2 .}
\end{aligned}
$$


Now,

$$
\begin{aligned}
& \prod 1=\prod_{p \in V\left(\Gamma_{1}\right)} \prod_{q_{1} q_{2} \in E\left(\Gamma_{2}\right)}\left[d\left(p, q_{1}\right)+d\left(p, q_{2}\right)\right] \\
& \leq \frac{1}{\mu_{1} \omega_{2}}\left[\sum_{p \in V\left(\Gamma_{1}\right)} \sum_{q_{1} q_{2} \in E\left(\Gamma_{2}\right)}\left[2 d_{\Gamma_{1}}(p)+d_{\Gamma_{2}}\left(q_{1}\right)+d_{\Gamma_{2}}\left(q_{2}\right)\right]\right]^{\mu_{1} \omega_{2}}=\frac{1}{\mu_{1} \omega_{2}}\left[4 \omega_{1} \omega_{2}+\mu_{1} M_{1}\left(\Gamma_{2}\right)\right]^{\mu_{1} \omega_{2}}, \\
& \prod 2=\prod_{q \in V\left(\Gamma_{2}\right)} \prod_{p_{1} p_{2} \in E\left(Q_{k}\left(\Gamma_{1}\right)\right)}\left[d\left(p_{1}, q\right)+d\left(p_{2}, q\right)\right]=q \in V\left(\Gamma_{2}\right) \prod_{\substack { q \in V\left(\Gamma_{2}\right) \\
\begin{subarray}{c}{p_{1} p_{2} \in E\left(Q_{k}\left(\Gamma_{1}\right)\right) \\
p_{1} \in V\left(\Gamma_{1}\right) \\
p_{1} p_{2} \in V\left(Q_{k}\left(\Gamma_{1}\right)\right)-\mu\left(\Gamma_{1}\right){ q \in V ( \Gamma _ { 2 } ) \\
\begin{subarray} { c } { p _ { 1 } p _ { 2 } \in E ( Q _ { k } ( \Gamma _ { 1 } ) ) \\
p _ { 1 } \in V ( \Gamma _ { 1 } ) \\
p _ { 1 } p _ { 2 } \in V ( Q _ { k } ( \Gamma _ { 1 } ) ) - \mu ( \Gamma _ { 1 } ) } }\end{subarray}}\left[d\left(p_{1}, q\right)+d\left(p_{2}, q\right)\right] \\
& \times \prod_{q \in V\left(\Gamma_{2}\right)} \prod_{\substack{p_{1} p_{2} \in E\left(Q_{k}\left(\Gamma_{1}\right)\right) \\
p_{1} p_{2} \in V\left(Q_{k}\left(\Gamma_{1}\right)\right)-\mu\left(\Gamma_{1}\right)}}\left[d\left(p_{1}, q\right)+d\left(p_{2}, q\right)\right] \\
& =\prod^{\prime} 2 \times \prod^{\prime \prime} 2 .
\end{aligned}
$$

\section{Consider}

$$
\begin{aligned}
& \prod^{\prime} 2=\prod_{q \in V\left(\Gamma_{2}\right)} \prod_{\substack{p_{1} p_{2} \in E\left(Q_{k}\left(\Gamma_{1}\right)\right) \\
p_{1} \in V\left(\Gamma_{1}\right) \\
p_{1} p_{2} \in V\left(Q_{k}\left(\Gamma_{1}\right)\right)-\mu\left(\Gamma_{1}\right)}}\left[d\left(p_{1}, q\right)+d\left(p_{2}, q\right)\right] \\
& \leq \frac{1}{2 \omega_{1} \mu_{2}}\left[\sum_{\substack{q \in V\left(\Gamma_{2}\right) \\
p_{1} p_{2} \in E\left(Q_{k}\left(\Gamma_{1}\right)\right) \\
p_{1} \in V\left(\Gamma_{1} \in V\left(Q_{k}\left(\Gamma_{1}\right)\right)-\mu\left(\Gamma_{1}\right)\right.}}\left[d_{Q_{k}\left(\Gamma_{1}\right)}\left(p_{1}\right)+d_{\Gamma_{2}}(q)+d_{Q_{k}\left(\Gamma_{1}\right)}\left(p_{2}\right)\right]\right]^{2 \omega_{1} \mu_{2}} \\
& =\frac{1}{2 \omega_{1} \mu_{2}}\left[\sum_{q \in V\left(\Gamma_{2}\right)} \sum_{p_{1} \in V\left(\Gamma_{1}\right)}\left[d_{\Gamma_{1}}\left(p_{1}\right)+d_{\Gamma_{2}}(q)\right]\right]^{2 \omega_{1} \mu_{2}}
\end{aligned}
$$

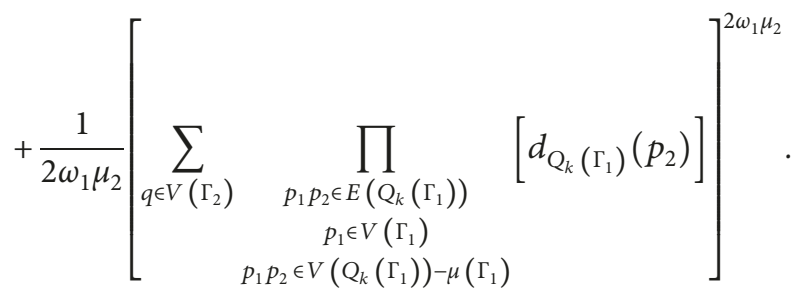

Then,

$$
\prod^{\prime} 2=\frac{1}{2 \omega_{1} \mu_{2}}\left[4 \omega_{1} \omega_{2}+3 \mu_{2} M_{1}\left(\Gamma_{1}\right)\right]^{2 \omega_{1} \mu_{2}} .
$$

$$
\prod^{\prime \prime} 2=\prod_{q \in V\left(\Gamma_{2}\right)} \prod_{\substack{p_{1} p_{2} \in E\left(Q_{k}\left(\Gamma_{1}\right)\right) \\ p_{1} p_{2} \in V\left(Q_{k}\left(\Gamma_{1}\right)\right)-\mu\left(\Gamma_{1}\right)}}\left[d\left(p_{1}, q\right)+d\left(p_{2}, q\right)\right] .
$$

Now we take 
Let $\prod^{\prime \prime} 2=\prod 3 \times \prod 4$, then

$$
\left.\begin{array}{rl}
\prod 3 & =\prod_{q \in V\left(\Gamma_{2}\right)} \prod_{\substack{p_{1} p_{2} \in E\left(Q_{k}\left(\Gamma_{1}\right)\right) \\
p_{1} p_{2} \in V\left(Q_{k}\left(\Gamma_{1}\right)\right)-\mu\left(\Gamma_{1}\right)}}\left[d_{Q_{k}\left(\Gamma_{1}\right)}\left(p_{1}\right)+d_{Q_{k}\left(\Gamma_{1}\right)}\left(p_{2}\right)\right] \\
& \leq \frac{1}{\omega_{1} \mu_{2}(k-1)}\left[2(k-1) \sum_{q \in V\left(\Gamma_{2}\right)} \sum_{u v \in E\left(\Gamma_{1}\right)}\left[d_{\Gamma_{1}}(u)+d_{\Gamma_{1}}(v)\right]\right]^{\omega_{1} \mu_{2}(k-1)} \\
& =\frac{1}{\omega_{1} \mu_{2}(k-1)}\left[2(k-1) \mu_{2} M_{1}\left(\Gamma_{1}\right)\right]^{\omega_{1} \mu_{2}(k-1)}, \\
\prod 4 & =\prod_{q \in V\left(\Gamma_{2}\right) \quad p_{1} p_{2} \in E\left(Q_{k}\left(\Gamma_{1}\right)\right)}\left[d_{Q_{k}\left(\Gamma_{1}\right)}\left(p_{1}\right)+d_{Q_{k}\left(\Gamma_{1}\right)}\left(p_{2}\right)\right] \\
& \leq \frac{1}{k \mu_{2}\left(2 \omega_{1}-\mu_{1}\right)}\left[\sum_{q \in V\left(\Gamma_{2}\right)} \sum_{p_{1} p_{2} \in E\left(Q_{k}\left(\Gamma_{1}\right)\right)}\left[d_{Q_{k}\left(\Gamma_{1}\right)}\left(p_{1}\right)+d_{Q_{k}\left(\Gamma_{1}\right)}\left(p_{2}\right)\right]\right.
\end{array}\right]
$$

Therefore, we have

$$
\begin{aligned}
\prod_{1}\left(\Gamma_{1}+{ }_{Q_{k}} \Gamma_{2}\right) & \leq \frac{1}{\mu_{1} \omega_{2}}\left[4 \omega_{1} \omega_{2}+\mu_{1} M_{1}\left(\Gamma_{2}\right)\right]^{\mu_{1} \omega_{2}} \times \frac{1}{2 \omega_{1} \mu_{2}}\left[4 \omega_{1} \omega_{2}+3 \mu_{2} M_{1}\left(\Gamma_{1}\right)\right]^{2 \omega_{1} \mu_{2}} \\
& \times \frac{1}{\omega_{1} \mu_{2}(k-1)}\left[2(k-1) \mu_{2} M_{1}\left(\Gamma_{1}\right)\right]^{\omega_{1} \mu_{2}(k-1)} \\
& \times \frac{1}{k \mu_{2}\left(2 \omega_{1}-\mu_{1}\right)}\left[(k) \mu_{2}\left[M_{3}\left(\Gamma_{1}\right)+2 M_{2}\left(\Gamma_{1}\right)-2 M_{1}\left(\Gamma_{1}\right)\right]\right]^{k \mu_{2}\left(2 \omega_{1}-\mu_{1}\right)}
\end{aligned}
$$

Next,

$$
\begin{aligned}
\prod_{2}\left(\Gamma_{1}+{ }_{Q_{k}} \Gamma_{2}\right) & =\prod_{\left(p_{1}, q_{1}\right)}\left[d\left(p_{2}, q_{2}\right) \in E\left(\Gamma_{1}+Q_{k} \Gamma_{2}\right)\right. \\
& =\prod_{p \in V\left(\Gamma_{1}\right)} \prod_{q_{1} q_{2} \in E\left(\Gamma_{2}\right)}\left[d\left(p, q_{1}\right) d\left(p, q_{2}\right)\right] \times \prod_{q \in V\left(\Gamma_{2}\right)} \prod_{p_{1} p_{2} \in E\left(Q_{k}\left(\Gamma_{1}\right)\right)}\left[d\left(p_{1}, q\right) d\left(p_{2}, q\right)\right] \\
& =\prod 1 \times \prod 2 .
\end{aligned}
$$

Consider 


$$
\begin{aligned}
\prod 1= & \prod_{p \in V\left(\Gamma_{1}\right)} \prod_{q_{1} q_{2} \in E\left(\Gamma_{2}\right)}\left[d\left(p, q_{1}\right) d\left(p, q_{2}\right)\right] \\
\leq & \frac{1}{\mu_{1} \omega_{2}}\left[\sum_{p \in V\left(\Gamma_{1}\right)} \sum_{q_{1} q_{2} \in E\left(\Gamma_{2}\right)}\left[d\left(p, q_{1}\right) d\left(p, q_{2}\right)\right]\right]^{\mu_{1} \omega_{2}} \\
= & \frac{1}{\mu_{1} \omega_{2}}\left[\omega_{2} M_{1}\left(\Gamma_{1}\right)+2 \omega_{1} M_{1}\left(\Gamma_{2}\right)+\mu_{1} M_{2}\left(\Gamma_{2}\right)\right]^{\mu_{1} \omega_{2}}, \\
\prod 2= & \prod_{q \in V\left(\Gamma_{2}\right)} \prod_{p_{1} p_{2} \in E\left(Q_{k}\left(\Gamma_{1}\right)\right)}\left[d\left(p_{1}, q\right) d\left(p_{2}, q\right)\right] \\
= & \prod_{q \in V\left(\Gamma_{2}\right)} \prod_{p_{1} p_{2} \in E\left(Q_{k}\left(\Gamma_{1}\right)\right)}\left[d\left(p_{1}, q\right) d\left(p_{2}, q\right)\right] \\
& \times \prod_{p_{1} \in V\left(\Gamma_{1}\right)}\left[d\left(p_{1}, q\right) d\left(p_{2}, q\right)\right] \\
= & \prod_{q \in V\left(\Gamma_{2}\right)} 2 \times \prod_{p_{1} p_{2} \in V\left(Q_{k}\left(\Gamma_{1}\right)\right)-\mu\left(\Gamma_{1}\right)} \prod_{2}^{\prime \prime} 2 .
\end{aligned}
$$

Then,

$$
\begin{aligned}
& \prod^{\prime} 2=\prod_{q \in V\left(\Gamma_{2}\right)} \prod_{\substack{p_{1} p_{2} \in E\left(Q_{k}\left(\Gamma_{1}\right)\right) \\
p_{1} \in V\left(\Gamma_{1}\right)}}\left[d\left(p_{1}, q\right) d\left(p_{2}, q\right)\right] \\
& \leq \frac{1}{2 \omega_{1} \mu_{2}}\left[\sum_{\substack{q \in V\left(\Gamma_{2}\right) \\
p_{1} p_{2} \in E\left(Q_{k}\left(\Gamma_{1}\right)\right) \\
p_{1} \in V\left(\Gamma_{1}\right)}}\left[d\left(p_{1}, q\right) d\left(p_{2}, q\right)\right]\right]^{2 \omega_{1} \mu_{2}} \\
& =\frac{1}{2 \omega_{1} \mu_{2}}\left[\sum_{q \in V\left(\Gamma_{2}\right)} \sum_{\substack{p_{1} p_{2} \in E\left(Q_{k}\left(\Gamma_{1}\right)\right) \\
p_{1} \in V\left(\Gamma_{1}\right)}}\left[d_{\Gamma_{1}}\left(p_{1}\right)+d_{\Gamma_{2}}(q)\right] d_{Q_{k}\left(\Gamma_{1}\right)}\left(p_{2}\right)\right]^{2 \omega_{1} \mu_{2}} \\
& =\frac{1}{2 \omega_{1} \mu_{2}}\left[4 \omega_{2} M_{1}\left(\Gamma_{1}\right)+\mu_{2}\left[M_{3}\left(\Gamma_{1}\right)+2 M_{2}\left(\Gamma_{1}\right)\right]\right]^{2 \omega_{1} \mu_{2}}, \\
& \prod^{\prime \prime} 2=\prod_{q \in V\left(\Gamma_{2}\right)} \prod_{\substack{p_{1} p_{2} \in E\left(Q_{k}\left(\Gamma_{1}\right)\right) \\
p_{1} p_{2} \in V\left(Q_{k}\left(\Gamma_{1}\right)\right)-\mu\left(\Gamma_{1}\right)}}\left[d\left(p_{1}, q\right) d\left(p_{2}, q\right)\right]
\end{aligned}
$$


Let $\prod^{\prime \prime} 2=\prod 3 \times \prod 4$, then

$$
\begin{aligned}
& \prod 3=\prod_{q \in V\left(\Gamma_{2}\right)} \prod_{\substack{p_{1} p_{2} \in E\left(Q_{k}\left(\Gamma_{1}\right)\right) \\
p_{1} p_{2} \in V\left(Q_{k}\left(\Gamma_{1}\right)\right)-\mu\left(\Gamma_{1}\right)}}\left[d_{Q_{k}\left(\Gamma_{1}\right)}\left(p_{1}\right) d_{Q_{k}\left(\Gamma_{1}\right)}\left(p_{2}\right)\right] \\
& \leq \frac{1}{(k-1) \mu_{2} \omega_{1}}\left[\sum_{q \in V\left(\Gamma_{2}\right)} \sum_{\substack{p_{1} p_{2} \in E\left(Q_{k}\left(\Gamma_{1}\right)\right) \\
p_{1} p_{2} \in V\left(Q_{k}\left(\Gamma_{1}\right)\right)-\mu\left(\Gamma_{1}\right)}}\left[d_{Q_{k}\left(\Gamma_{1}\right)}\left(p_{1}\right) d_{Q_{k}\left(\Gamma_{1}\right)}\left(p_{2}\right)\right]\right]^{(k-1) \mu_{2} \omega_{1}} \\
& =\frac{1}{(k-1) \mu_{2} \omega_{1}}\left[(k-1) \mu_{2}\left[2 M_{2}\left(\Gamma_{1}\right)+M_{3}\left(\Gamma_{1}\right)\right]\right]^{(k-1) \mu_{2} \omega_{1}}, \\
& \prod 4=\prod_{q \in V\left(\Gamma_{2}\right)} \prod_{\substack{p_{1} p_{2} \in E\left(Q_{k}\left(\Gamma_{1}\right)\right) \\
p_{1} p_{2} \in V\left(Q_{k}\left(\Gamma_{1}\right)\right)-\mu\left(\Gamma_{1}\right)}}\left[d_{Q_{k}\left(\Gamma_{1}\right)}\left(p_{1}\right) d_{Q_{k}\left(\Gamma_{1}\right)}\left(p_{2}\right)\right]
\end{aligned}
$$

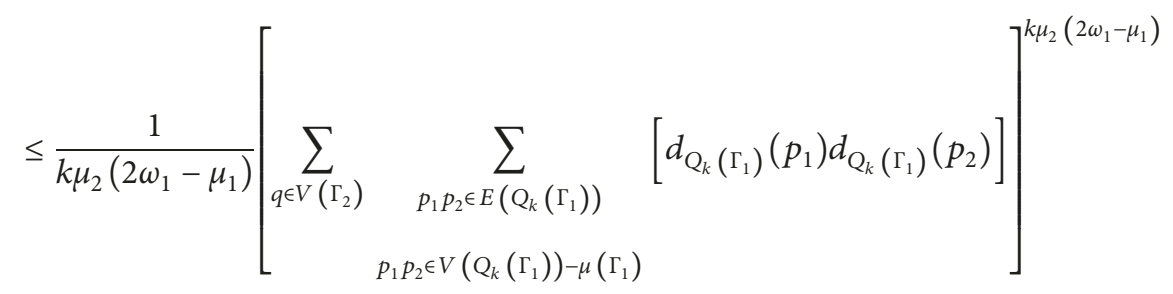

$$
\begin{aligned}
& =\frac{1}{k \mu_{2}\left(2 \omega_{1}-\mu_{1}\right)}\left[(k) \sum_{q \in V\left(\Gamma_{2}\right)} \sum_{\substack{u v \in E\left(\Gamma_{1}\right) \\
v w \in E\left(\Gamma_{2}\right)}}\left[d_{\Gamma_{1}}(u)+d_{\Gamma_{1}}(v)\right]\left[d_{\Gamma_{1}}(v)+d_{\Gamma_{1}}(w)\right]\right]^{k \mu_{2}\left(2 \omega_{1}-\mu_{1}\right)} \\
& =\frac{1}{k \mu_{2}\left(2 \omega_{1}-\mu_{1}\right)}\left[(k) \mu_{2}\left[\frac{1}{2} M_{4}\left(\Gamma_{1}\right)-\frac{1}{2} M_{3}\left(\Gamma_{1}\right)\right]+\sum_{u v \in V\left(\Gamma_{1}\right)} r d_{\Gamma_{1}}(u) d_{\Gamma_{1}}(v)\right]^{k \mu_{2}\left(2 \omega_{1}-\mu_{1}\right)} \\
& +\frac{1}{k \mu_{2}\left(2 \omega_{1}-\mu_{1}\right)}\left[\sum_{v \in V\left(\Gamma_{1}\right)} d_{\Gamma_{1}}^{2}(v) \sum_{\substack{u \in V\left(\Gamma_{1}\right) \\
u v \in E\left(\Gamma_{1}\right)}} d_{\Gamma_{1}}(u)-2 M_{2}\left(\Gamma_{1}\right)\right]^{k \mu_{2}\left(2 \omega_{1}-\mu_{1}\right)},
\end{aligned}
$$


where $r$ is the total neighbors that we consider mutual nodes

of $u$ and $v$ in $\left(\Gamma_{1}\right)$.

Therefore,

$$
\begin{aligned}
\prod_{2}\left(\Gamma_{1}+{ }_{Q^{k}} \Gamma_{2}\right) \leq & \frac{1}{\mu_{1} \omega_{2}}\left[\omega_{2} M_{1}\left(\Gamma_{1}\right)+2 \omega_{1} M_{1}\left(\Gamma_{2}\right)+\mu_{1} M_{2}\left(\Gamma_{2}\right)\right]^{\mu_{1} \omega_{2}} \\
& \times \frac{1}{2 \mu_{2} \omega_{1}}\left[4 \omega_{2} M_{1}\left(\Gamma_{1}\right)+\mu_{2}\left[M_{3}\left(\Gamma_{1}\right)+2 M_{2}\left(\Gamma_{1}\right)\right]\right]^{2 \mu_{2} \omega_{1}} \\
& \times \frac{1}{(k-1) \mu_{2} \omega_{1}}\left[(k-1) \mu_{2}\left[2 M_{2}\left(\Gamma_{1}\right)+M_{3}\left(\Gamma_{1}\right)\right]\right]^{(k-1) \mu_{2} \omega_{1}} \\
& \times \frac{1}{k \mu_{2}\left(2 \omega_{1}-\mu_{1}\right)}\left[(k) \mu_{2}\left[\frac{1}{2} M_{4}\left(\Gamma_{1}\right)-\frac{1}{2} M_{3}\left(\Gamma_{1}\right)\right]+\sum_{u v \in V\left(\Gamma_{1}\right)} r d_{\Gamma_{1}}(u) d_{\Gamma_{1}}(v)\right]^{k \mu_{2}\left(2 \omega_{1}-\mu_{1}\right)} \\
& +\frac{1}{k \mu_{2}\left(2 \omega_{1}-\mu_{1}\right)}\left[\sum_{v \in V\left(\Gamma_{1}\right)} d_{\left.\Gamma_{1}(v) \sum_{\substack{u \in V\left(\Gamma_{1}\right) \\
u v E\left(\Gamma_{1}\right)}} d_{\Gamma_{1}}(u)-2 M_{2}\left(\Gamma_{1}\right)\right]^{k \mu_{2}\left(2 \omega_{1}-\mu_{1}\right)}}\right.
\end{aligned}
$$

Theorem 4. Let $\Gamma_{1}, \Gamma_{2}$ be two graphs with $\left|V\left(\Gamma_{1}\right)\right|=\mu_{1}$, $\left|V\left(\Gamma_{2}\right)\right|=\mu_{2},\left|E\left(\Gamma_{1}\right)\right|=\omega_{1}$, and $\left|E\left(\Gamma_{2}\right)\right|=\omega_{2}$. For $\mu_{1}, \mu_{2} \geq 4$ and and $k \geq 1$, we have

$$
\begin{aligned}
& \prod_{1}\left(\Gamma_{1}+{ }_{T_{k}} \Gamma_{2}\right) \leq \frac{1}{\mu_{1} \omega_{2}}\left[\mu_{1} M_{1}\left(\Gamma_{2}\right)+8 \omega_{1} \omega_{2}\right]^{\mu_{1} \omega_{2}} \times \frac{1}{\omega_{1} \mu_{2}}\left[4 \omega_{1} \omega_{2}+2 \mu_{2} M_{1}\left(\Gamma_{1}\right)\right]^{\omega_{1} \mu_{2}} \\
& \times \frac{1}{2 \omega_{1} \mu_{2}}\left[4 \omega_{1} \omega_{2}+4 \mu_{2} M_{1}\left(\Gamma_{1}\right)\right]^{2 \omega_{1} \mu_{2}} \times \frac{1}{\omega_{1} \mu_{2}(k-1)}\left[2(k-1) \mu_{2} M_{1}\left(\Gamma_{1}\right)\right]^{\omega_{1} \mu_{2}(k-1)} \\
& \times \frac{1}{k \mu_{2}\left(2 \omega_{1}-\mu_{1}\right)}\left[(k) \mu_{2}\left[M_{3}\left(\Gamma_{1}\right)+2 M_{2}\left(\Gamma_{1}\right)-2 M_{1}\left(\Gamma_{1}\right)\right]\right]^{k \mu_{2}\left(2 \omega_{1}-\mu_{1}\right)}, \\
& \prod_{2}\left(\Gamma_{1}+T_{k} \Gamma_{2}\right) \leq \frac{1}{\omega_{2} \mu_{1}}\left[4 \omega_{2} M_{1}\left(\Gamma_{1}\right)+4 \omega_{1} M_{1}\left(\Gamma_{2}\right)+\mu_{1} M_{2}\left(\Gamma_{2}\right)\right]^{\omega_{2} \mu_{1}} \times \frac{1}{\omega_{1} \mu_{2}}\left[4 \mu_{2} M_{2}\left(\Gamma_{1}\right)+4 \omega_{2} M_{1}\left(\Gamma_{1}\right)+\omega_{1} M_{1}\left(\Gamma_{2}\right)\right]^{\omega_{1} \mu_{2}} \\
& \times \frac{1}{2 \mu_{2} \omega_{1}}\left[4 \omega_{2} M_{1}\left(\Gamma_{1}\right)+\mu_{2}\left[2 M_{3}\left(\Gamma_{1}\right)+4 M_{2}\left(\Gamma_{1}\right)\right]\right]^{2 \mu_{2} \omega_{1}} \times \frac{1}{(k-1) \mu_{2} \omega_{1}}\left[(k-1) \mu_{2}\left[2 M_{2}\left(\Gamma_{1}\right)+M_{3}\left(\Gamma_{1}\right)\right]\right]^{(k-1) \mu_{2} \omega_{1}} \\
& \times \frac{1}{k \mu_{2}\left(2 \omega_{1}-\mu_{1}\right)}\left[(k) \mu_{2}\left[\frac{1}{2} M_{4}\left(\Gamma_{1}\right)-\frac{1}{2} M_{3}\left(\Gamma_{1}\right)\right]+\sum_{u v \in V\left(\Gamma_{1}\right)}^{\left.r d_{\Gamma_{1}}(u) d_{\Gamma_{1}}(v)\right]}\right] \\
&+\frac{1}{k \mu_{2}\left(2 \omega_{1}-\mu_{1}\right)}\left[\sum_{v \in V\left(\Gamma_{1}\right)}^{k \mu_{2}\left(2 \omega_{1}-\mu_{1}\right)}\right] \\
& d_{\Gamma_{1}}^{2}(v) \sum_{u \in V\left(\Gamma_{1}\right)} d_{\Gamma_{1}}(u)-2 \omega_{2}\left(\Gamma_{1}\right) \\
& u v \in E\left(\Gamma_{1}\right)
\end{aligned}
$$




\section{Conclusion}

For $a, b \geq 4$ and $k=4$, we consider that $\Gamma_{1}=P_{a}$ and $\Gamma_{2}=P_{b}$ are specific examples of alkane known as paths having orders $a$ and $b$, respectively. Then, the following results are the direct outcomes of the above four theorems.

$$
\begin{aligned}
& \prod_{1}\left(P_{a+S_{4}} P_{b}\right)=\frac{1}{a b-a}[8 a b-10 a-4 a+4]^{a b-a} \times \frac{1}{2 a b-2 b}[12 a b-4 a-14 b+4]^{2 a b-2 b} \times \frac{1}{3 a b-3 b}[12 a b-12 b]^{3 a b-3 b}, \\
& \prod_{2}\left(P_{a+S_{4}} P_{b}\right)=\frac{1}{a b-a}[16 a b-24 a-14 b+18]^{a b-a} \times \frac{1}{2 a b-2 b}[16 a b-8 a-20 b+8]^{2 a b-2 b} \times \frac{1}{3 a b-3 b}[12 a b-12 b]^{3 a b-3 b}, \\
& \prod_{1}\left(P_{a+R_{4}} P_{b}\right)=\frac{1}{a b-a}[12 a b-14 a-8 b+8]^{a b-a} \times \frac{1}{a b-b}[12 a b-4 a-16 b+4]^{a b-b} \\
& \times \frac{1}{2 a b-2 b}[16 a b-4 a-20 b+4]^{2 a b-2 b} \times \frac{1}{3 a b-3 b}[12 a b-12 b]^{3 a b-3 b} \\
& \prod_{2}\left(P_{a+R_{4}} P_{b}\right)=\frac{1}{a b-a}[36 a b-48 a-40 b+48]^{a b-a} \times \frac{1}{a b-b}[36 a b-22 a-60 b+30]^{a b-b} \\
& \times \frac{1}{2 a b-2 b}[24 a b-8 a-32 b+8]^{2 a b-2 b} \times \frac{1}{3 a b-3 b}[12 a b-12 b]^{3 a b-3 b} \\
& \prod_{1}\left(P_{a+Q_{4}} P_{b}\right)=\frac{1}{a b-a}[8 a b-10 a-4 b+4]^{a b-a} \times \frac{1}{2 a b-2 b}[16 a b-4 a-22 b+4]^{2 a b-2 b} \\
& \times \frac{1}{3 a b-3 b}[24 a b-36 b]^{3 a b-3 b} \times \frac{1}{4 a b-8 b}[32 a b-72 b]^{4 a b-8 b}, \\
& \prod_{2}\left(P_{a+Q_{4}} P_{b}\right)=\frac{1}{a b-a}[16 a b-24 a-14 b+18]^{a b-a} \times \frac{1}{2 a b-2 b}[32 a b-16 a-54 b+24]^{2 a b-2 b} \\
& \times \frac{1}{3 a b-3 b}[48 a b-90 b]^{3 a b-3 b} \times \frac{1}{4 a b-8 b}[64 a b-160 b]^{4 a b-8 b}, \\
& \prod_{1}\left(P_{a+T_{4}} P_{b}\right)=\frac{1}{a b-a}[12 a b-14 a-8 b+8]^{a b-a} \times \frac{1}{a b-b}[12 a b-4 a-16 b+4]^{a b-b} \\
& \times \frac{1}{2 a b-2 b}[20 a b-4 a-28 b+4]^{2 a b-2 b} \times \frac{1}{4 a b-8 b}[24 a b-36 b]^{4 a b-8 b} \times \frac{1}{3 a b-3 b}[32 a b-72 b]^{3 a b-3 b}, \\
& \prod_{2}\left(P_{a+T_{4}} P_{b}\right)=\frac{1}{a b-a}[36 a b-48 a-40 b+48]^{a b-a} \times \frac{1}{a b-b}[36 a b-22 a-60 b+30]^{a b-b} \\
& \times \frac{1}{2 a b-2 b}[48 a b-16 a-84 b+24]^{2 a b-2 b} \times \frac{1}{4 a b-8 b}[48 a b-90 b]^{4 a b-8 b} \times \frac{1}{3 a b-3 b}[64 a b-160 b]^{3 a b-3 b},
\end{aligned}
$$


In this paper, for the integer $k \geq 1$, we computed upper bonds of the 1st and 2nd multiplicative Zagreb indices of the generalized F-sum graphs $\left[\left(\Gamma_{1+F_{k}} \Gamma_{2}\right)\right.$ for $F_{k} \in\left\{S_{k}, R_{k}, Q_{k}\right.$, $\left.\left.\& T_{k}\right\}\right]$ that was defined by Liu et al. [30]. However, the problem is still open to compute the other topological indices of the generalized F-sum graphs.

\section{Data Availability}

The data used to support the findings of this study are cited at relevant places within the text as references.

\section{Conflicts of Interest}

The authors declare no conflicts of interest.

\section{Authors' Contributions}

All authors contributed equally to this work.

\section{Acknowledgments}

This research was supported by the Applied Basic Research (Key Project) of Sichuan Province under grant 2017JY0095 and the Soft Science Project of Sichuan Province under grant 2017 ZR0041.

\section{References}

[1] G. Rücker and C. Rücker, "On topological indices, boiling points, and cycloalkanes," Journal of Chemical Information and Computer Sciences, vol. 39, no. 5, pp. 788-802, 1999.

[2] M. Randić, "On characterization of molecular branching," Journal of the American Chemical Society, vol. 97, pp. 66096615, 1975.

[3] A. R. Matamala and E. Estrada, "Generalised topological indices: optimisation methodology and physico-chemical interpretation," Chemical Physics Letters, vol. 410, no. 4-6, pp. 343-347, 2005.

[4] W. Yan, B.-Y. Yang, and Y.-N. Yeh, "The behavior of Wiener indices and polynomials of graphs under five graph decorations," Applied Mathematics Letters, vol. 20, no. 3, pp. 290-295, 2007.

[5] H. González-Dìaz, S. Vilar, L. Santana, and E. Uriarte, "Medicinal chemistry and bioinformatics-current trends in drugs discovery with networks topological indices," Current Topics in Medicinal Chemistry, vol. 7, no. 10, pp. 1015-1029, 2007.

[6] X. Li and J. Zheng, "A unified approach to the extremal trees for different indices," MATCH-Communications in Mathematical and in Computer Chemistry, vol. 54, pp. 195-208, 2005.

[7] J.-B. Liu, C. Wang, S. Wang, and B. Wei, "Zagreb indices and multiplicative Zagreb indices of eulerian graphs," Bulletin of the Malaysian Mathematical Sciences Society, vol. 42, no. 1, pp. 67-78, 2019.

[8] R. Todeschini and V. Consonni, "New local vertex invariants and molecular descriptors based on functions of the vertex degrees," MATCH-Communications in Mathematical and in Computer Chemistry, vol. 64, pp. 359-372, 2010.

[9] I. Gutman and B. Furtula, Novel Molecular Structure Descriptors-Theory and Applications, University of Kragujevac, Kragujevac, Serbia, 2010.
[10] Z. Shao, M. Siddiqui, and M. Muhammad, "Computing Zagreb indices and Zagreb polynomials for symmetrical nanotubes," Symmetry, vol. 10, no. 7, pp. 244-254, 2018.

[11] M. Eliasi, I. Gutman, and A. Iranmanesh, "Multiplicative versions of first Zagreb index," MATCH-Communications in Mathematical and in Computer Chemistry, vol. 68, pp. 217230, 2012.

[12] M. Eliasi and D. Vukičević, "Comparing the multiplicative Zagreb indices," Communications in Mathematical and in Computer Chemistry, vol. 69, pp. 765-773, 2013.

[13] F. Yan, Q. Shang, S. Xia, Q. Wang, and P. Ma, "Application of topological index in predicting ionic liquids densities by the quantitative structure property relationship method," Journal of Chemical \& Engineering Data, vol. 60, no. 3, pp. 734-739, 2015.

[14] M. V. Diudea, QSPR/QSAR Studies by Molecular Descriptors, NOVA, New York, NY, USA, 2001.

[15] J. Devillers and A. T. Balaban, Topological Indices and Related Descriptors in QSAR and QSPR, Gordon \& Breach, Amsterdam, Netherlands, 1999.

[16] R. Gozalbes, J. Doucet, and F. Derouin, "Application of topological descriptors in QSAR and drug design: history and new trends," Current Drug Target-Infectious Disorders, vol. 2, no. 1, pp. 93-102, 2002.

[17] J.-B. Liu, J. Zhao, and Z. Zhu, "On the number of spanning trees and normalized Laplacian of linear octagonal-quadrilateral networks," International Journal of Quantum Chemistry, vol. 119, Article ID e25971, 2019.

[18] H. Wiener, "Structural determination of paraffin boiling points," Journal of the American Chemical Society, vol. 69, no. 1, pp. 17-20, 1947.

[19] I. Gutman and N. Trinajstić, "Graph theory and molecular orbitals. Total $\varphi$-electron energy of alternant hydrocarbons," Chemical Physics Letters, vol. 17, no. 4, pp. 535-538, 1972.

[20] R. Todeschini, V. Consonni, R. Mannhold, H. Kubinyi, and H. Timmerman, Handbook of Molecular Descriptors, Wiley VCH, Weinheim, Germany, 2002.

[21] I. Gutman and O. Polansky, Mathematical Concepts in Organic Chemistry, Springer-Verlag, Berlin, Germany, 1986.

[22] I. Gutman, "Degree-based topological indices," Croatica Chemica Acta, vol. 86, no. 4, pp. 351-361, 2013.

[23] Z. Shao, M. Liang, and X. Xu, "Some new optimal generalized Sidon sequences," Ars Combinatoria, vol. 107, pp. 369-378, 2012.

[24] D. M. Cvetkocić, M. Doob, and H. Sachs, Spectra of Graphs: Theory and Application, Academic Press, New York, NY, USA, 1980.

[25] M. Eliasi and B. Taeri, "Four new sums of graphs and their Wiener indices," Discrete Applied Mathematics, vol. 157, no. 4, pp. 794-803, 2009.

[26] H. Deng, D. Sarala, S. K. Ayyaswamy, and S. Balachandran, "The Zagreb indices of four operations on graphs," Applied Mathematics and Computation, vol. 275, pp. 422-431, 2016.

[27] S. Akhter and M. Imran, "Computing the forgotten topological index of four operations on graphs," AKCE International Journal of Graphs and Combinatorics, vol. 14, no. 1, pp. 70-79, 2017.

[28] G. H. Shirdel, H. Rezapour, and A. M. Sayadi, "Hyper-Zagreb index of graph operations," Iranian Journal of Mathematical Chemistry, vol. 4, no. 2, pp. 213-220, 2013.

[29] J.-B. Liu, S. Javed, M. Javaid, and K. Shabbir, "Computing first general Zagreb index of operations on graphs," IEEE Access, vol. 7, pp. 47494-47502, 2019. 
[30] J.-B. Liu, M. Javaid, and H. M. Awais, "Computing Zagreb indices of the subdivision-related generalized operations of graphs," IEEE Access, vol. 7, pp. 105479-105488, 2019.

[31] M. Knor, R. Škrekovski, and A. Tepeh, "Convexity result and trees with large Balaban index," Applied Mathematics and Nonlinear Sciences, vol. 3, no. 2, pp. 433-446, 2018.

[32] Z. Shao, P. Wu, X. Zhang, D. Dimitrov, and J.-B. Liu, "On the maximum $A B C$ index of graphs with prescribed size and without pendent vertices," IEEE Access, vol. 6, pp. 604-616, 2018.

[33] Z. Shao, P. Wu, Y. Gao, I. Gutman, and X. Zhang, "On the maximum ABC index of graphs without pendent vertices," Applied Mathematics and Computation, vol. 315, pp. 298-312, 2017.

[34] J. A. Aledo, L. G. Diaz, S. Martinez, and J. C. Valverde, "Predecessors and Gardens of Eden in sequential dynamical systems over directed graphs," Applied Mathematics and Nonlinear Sciences, vol. 3, no. 2, pp. 593-602, 2018.

[35] J.-B. Liu, X.-F. Pan, F.-T. Hu, and F.-F. Hu, "Asymptotic Laplacian-energy-like invariant of lattices," Applied Mathematics and Computation, vol. 253, pp. 205-214, 2015.

[36] J.-B. Liu and X.-F. Pan, "Minimizing Kirchhoffindex among graphs with a given vertex bipartiteness," Applied Mathematics and Computation, vol. 291, p. 8488, 2016.

[37] D. Liu, C. Wang, and S. Wang, "Hamilton-connectivity of interconnection networks modeled by a product of graphs," Applied Mathematics and Nonlinear Sciences, vol. 3, no. 2, pp. 419-426, 2018.

[38] S. Wang, Z. Shao, J.-B. Liu, and B. Wei, "The bounds of vertex Padmakar-Ivan index on k-trees," Mathematics, vol. 7, no. 4, pp. 324-334, 2019.

[39] B. Basavanagoud, W. Gao, S. Patil, V. R. Desai, K. G. Mirajkar, and B. Pooja, "Computing first Zagreb index and F-index of new C-products of graphs," Applied Mathematics and Nonlinear Sciences, vol. 2, no. 1, pp. 285-298, 2017.

[40] M. Azari and A. Iranmanesh, "Some inequalities for the multiplicative sum Zagreb index of graph operations," Journal of Mathematical Inequalities, vol. 9, no. 3, pp. 727-738, 2015.

[41] K. C. Das, A. Yurttas, M. Togan, A. S. Cevik, and I. N. Cangul, "The multiplicative Zagreb indices of graph operations," Journal of Inequality and Applications, vol. 90, pp. 1-14, 2013.

[42] J. Liu and Q. Zhang, "Sharp upper bounds for multiplicative Zagreb indices," MATCH-Communications in Mathematical and in Computer Chemistry, vol. 68, pp. 231-240, 2012.

[43] T. Réti and I. Gutman, "Relations between ordinary and multiplicative Zagreb indices," Bulletin of International Mathematical Virtual Institute, vol. 2, pp. 133-140, 2012.

[44] S. Wang and B. Wei, "Multiplicative Zagreb indices of k-tree," Discrete Applied Mathematics, vol. 180, pp. 168-175, 2015.

[45] K. Xu and K. C. Das, "Trees, unicyclic and bicyclic graphs extremal with respect to multiplicative sum Zagreb index," MATCH-Communications in Mathematical and in Computer Chemistry, vol. 68, pp. 257-272, 2012.

[46] K. Xu and H. Hua, "A unified approach to extremal multiplicative Zagreb indices for trees, unicyclic and bicyclic graphs," MATCH-Communications in Mathematical and in Computer Chemistry, vol. 68, pp. 241-256, 2012. 

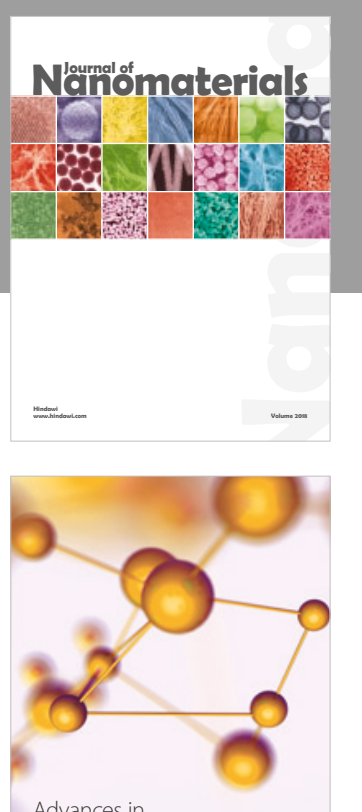

Physical Chemistry
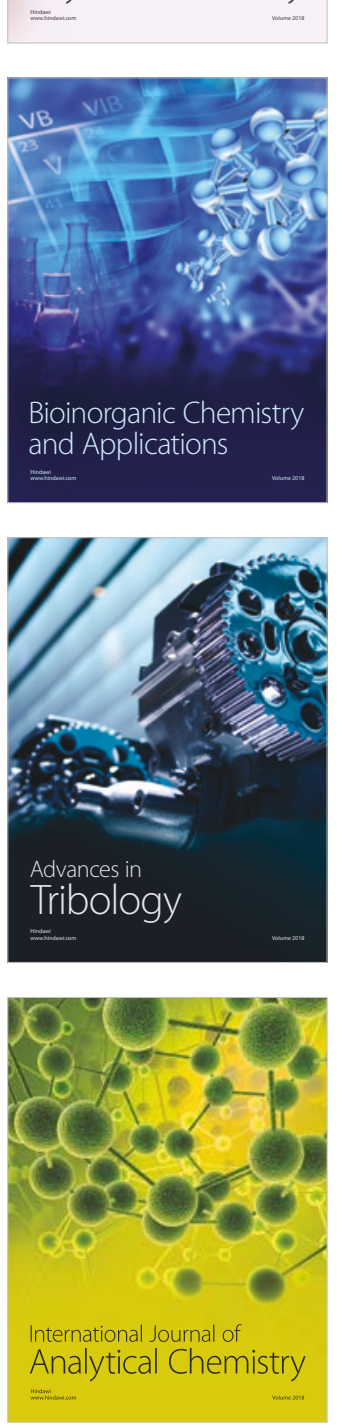

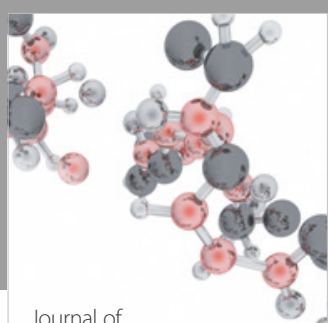

Analytical Methods

in Chemistry

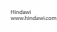

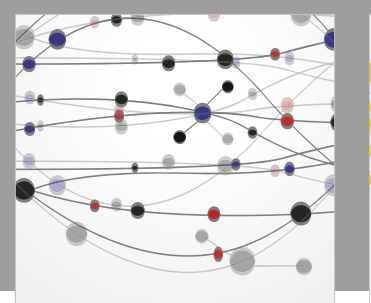

The Scientific World Journal

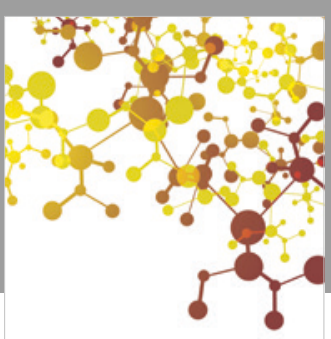

Journal of

Applied Chemistry
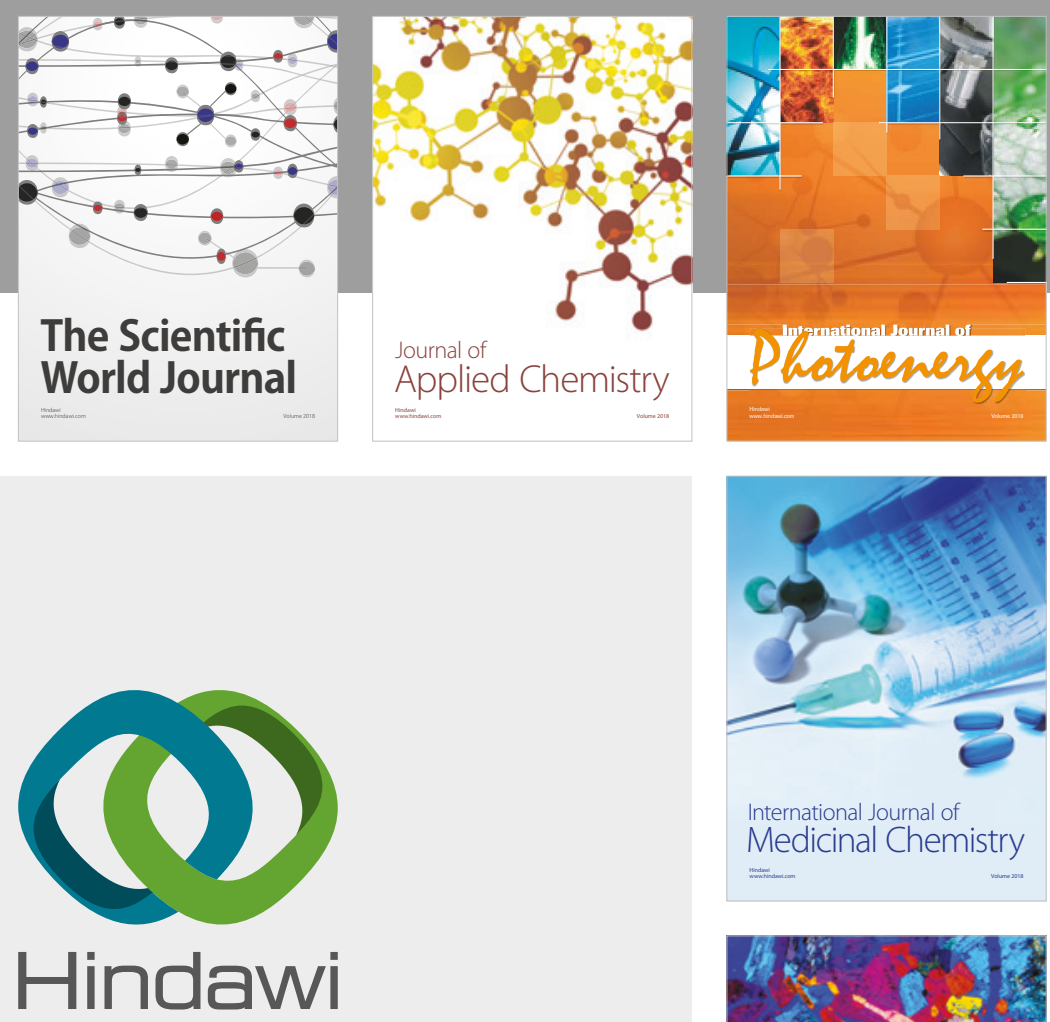

Submit your manuscripts at

www.hindawi.com
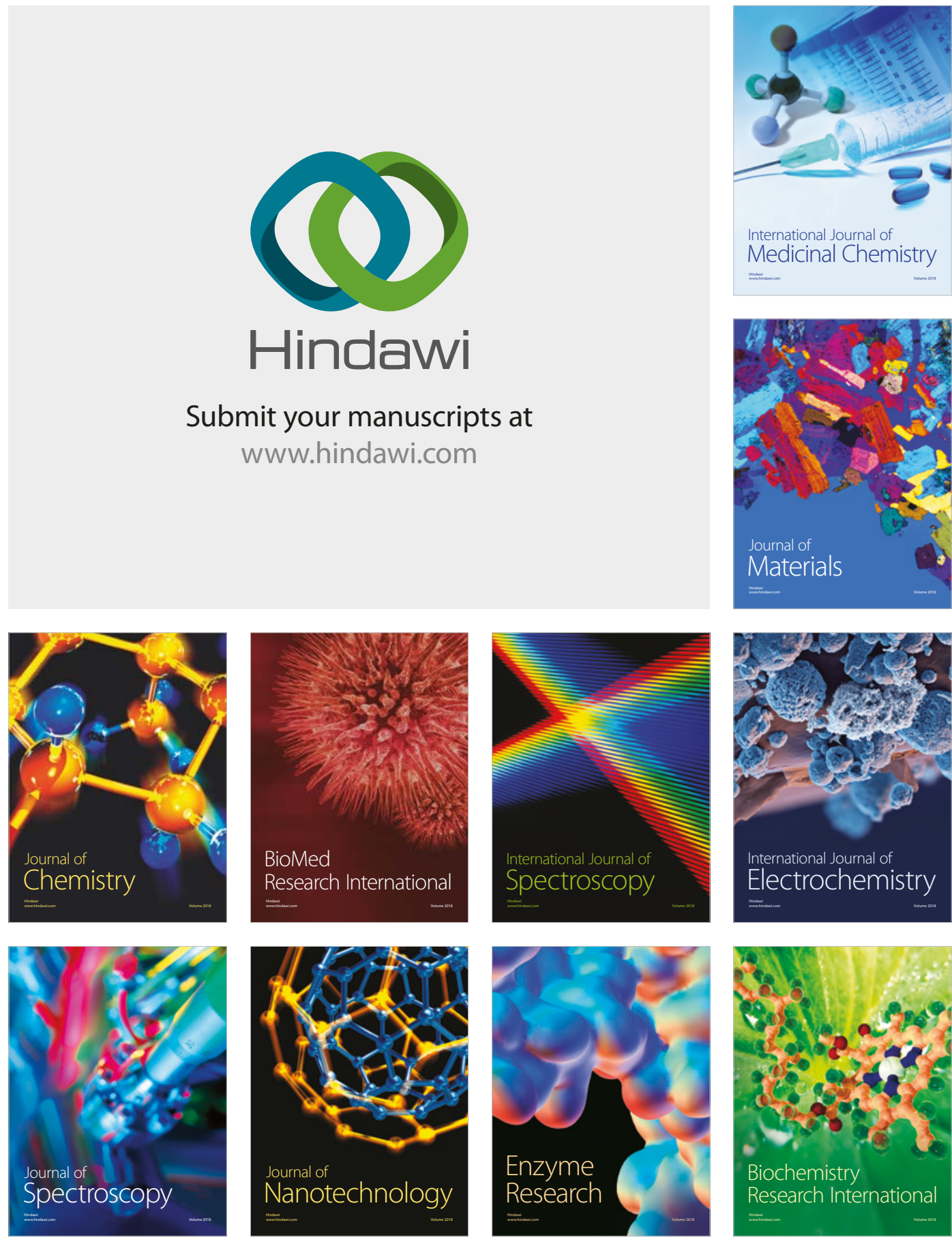
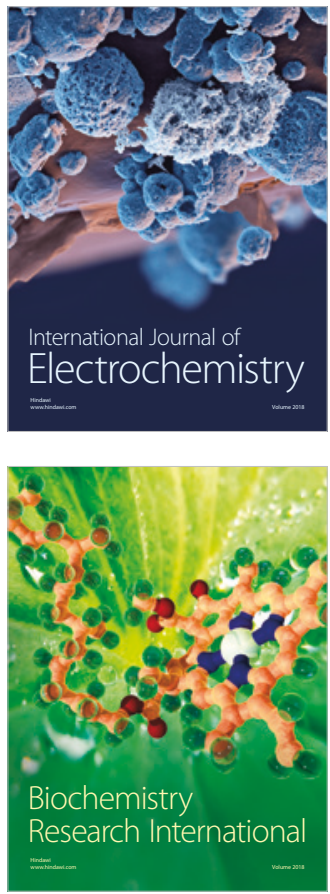